\title{
General soliton solutions to a coupled Fokas-Lenells equation
}

\author{
Liming Ling ${ }^{1}$, Bao-Feng Feng ${ }^{2}$, and Zuonong Zhu ${ }^{3}$ \\ 1 School of Mathematics, South China University of Technology, Guangzhou 510640, China \\ 2 School of Mathematical and Statistical Sciences, \\ The University of Texas Rio Grande Valley, Edinburg Texas, 78541, USA and \\ 3 School of Mathematical Sciences, Shanghai Jiaotong University, Shanghai, China
}

\begin{abstract}
In this paper, we firstly establish the multi-Hamiltonian structure and infinite many conservation laws for the vector Kaup-Newell hierarchy of the positive and negative orders. The first nontrivial negative flow corresponds to a coupled Fokas-Lenells equation. By constructing a generalized Darboux transformation and using a limiting process, all kinds of one-soliton solutions are constructed including the bright-dark soliton, the dark-anti-dark soliton and the breather-like solutions. Furthermore, multi-bright and multi-dark soliton solutions are derived and their asymptotic behaviors are investigated.

Keywords: Coupled Fokas-Lenells equation; Tu scheme; Multi-Hamiltonian structure; Generalized Darboux transformation; bright-dark soliton; dark-anti-dark soliton Mathematics Subject Classification: 39A10, 35Q58

PACS numbers: 05.45.Yv, 42.65.Tg, 42.81.Dp
\end{abstract}

\section{INTRODUCTION}

It is well known that the integrable nonlinear Schrödinger type equations, such as the classical nonlinear Schrödinger (NLS) equation [1], derivative-type NLS equation [2 -4], play an important role in the study of nonlinear wave propagation. Recently, a new integrable model, called the Fokas-Lenells (FL) equation [5, 6], was proposed in mono-mode optical fibers when certain higher order nonlinear effects were taken into account. The Hamiltonian structure and inverse scattering transformation for the FL equation were established by Fokas and Lenells in their original paper [5]. The algebraic geometry solution was construct in [7]. The rogue wave solutions for this system were constructed by several authors [8, 9]. It is interesting that, unlike the NLS equation, the FL equation admits both the bright and dark soliton solutions without the sign change of nonlinear term. The bright soliton solutions were constructed by Hirota's bilinear method [10], while the dark soliton solutions were constructed by Hirota's bilinear method [11] and by Bäcklund transformation [12], respectively.

As pointed out by Lenells, the FL equation is related to the derivative NLS equation. As a matter of fact, there are lots of studies regarding the derivative NLS equation such as the Hamiltonian structure 17], the inverse scattering method [14 16], the Darboux/Bäcklund transformation [18 22] and infinite many conservation laws [23, 24]. Meanwhile, there are quite a few works for the study of multi-component derivative NLS equations such as the Lax pair and its integrable properties [25 27] and exact solvable methods [28 30]. Similar to the case of the NLS equation [31], it is necessary to consider the two-component or multi-component generalizations of the FL equation for describing the effects of polarization or anisotropy. Most recently, the coupled FL equation has been studied by several authors [13, 28, 32].

Darboux transformation(DT) is a useful method to construct exact solutions for the integrable system 33 35]. Actually, the DT is a method related to the inverse scattering method which can be used to solve the initial value problem of integrable equations. Recently, there are some progresses to use the DT to construct some more general analytical solutions for NLS-type equations [30, 36 40]. However, in certain physical situations, two or more wave packets of different carrier frequencies appear simultaneously, and their interactions are governed by the coupled equations. Thus, a natural question is how to construct the generalized Darboux transformation and apply it to find exact solutions to the coupled integrable equations.

In this paper, we consider a coupled Fokas-Lenells equation

$$
\begin{gathered}
u_{1, x t}+u_{1}+\mathrm{i}\left(\left|u_{1}\right|^{2}+\frac{1}{2} \sigma\left|u_{2}\right|^{2}\right) u_{1, x}+\frac{\mathrm{i}}{2} \sigma u_{1} u_{2}^{*} u_{2, x}=0, \\
u_{2, x t}+u_{2}+\mathrm{i}\left(\sigma\left|u_{2}\right|^{2}+\frac{1}{2}\left|u_{1}\right|^{2}\right) u_{2, x}+\frac{\mathrm{i}}{2} u_{2} u_{1}^{*} u_{1, x}=0
\end{gathered}
$$

where $\sigma= \pm 1$. This equation was first proposed by Guo and Ling [28] with the matrix generalization of Lax pair. In [32], this coupled FL equation was reconstructed by the spectral gradient method. Zhang et. al constructed the bright, breather and first-order rogue wave solutions to a coupled FL equation, which is shown in [28] to be equivalent to the coupled FL equation (1) via a gauge transformation. Since the FL equation admits both the bright and dark 
soliton solution simultaneously, the structure of the soliton solutions to the coupled FL equation (1) is expected to be more complicated. It is the main objective of the present paper to explore the rich structure of soliton solutions to the coupled FL equation.

The Tu scheme [41 43] is an important technique to construct the Hamiltonian structure for the integrable hierarchy. In section II. we will apply the $\mathrm{Tu}$ scheme to construct the multi-Hamiltonian structure and the infinite many conservation laws for the coupled FL equation. First, Hamiltonian structures for the vector Kaup-Newell spectral problem involving both the positive and negative hierarchy are constructed. The first negative flow of the hierarchy is nothing but the coupled FL equation. Based on the Lax pair, the infinite many conservation laws are constructed in both positive and negative orders. In section III, we construct a generalized DT for the coupled FL equation (11). Then the general soliton solution to the coupled FL equation is constructed by the DT method. In section $\mathrm{V}$ based on the general soliton solution, a variety of single soliton solution from the zero and plane wave seed solutions are constructed and classified. These solutions include the bright soliton, the bright-dark soliton, the bright-anti-dark soliton, the dark-dark (D-D) soliton, the dark-anti-dark (D-AD) soliton, the anti-dark-anti-dark (AD-AD) soliton, the rational D-D/D-AD/AD-AD soliton, and the breather-like solution with nonzero boundary conditions. In section VI. we construct the multi-bright and multi-dark/anti-dark soliton solutions and perform its asymptotical analysis. The paper is concluded in Section VII by a brief summary and discussions.

\section{HAMILTONIAN STRUCTURE AND CONSERVATION LAWS FOR THE MULTI-COMPONENT KAUP-NEWELL SPECTRAL PROBLEM}

It is well known that the important criterions for the integrable hierarchy are multi-Hamiltonian structure and the infinitely many conservation laws. Firstly, we consider the Hamiltonian structure for the multi-component KaupNewell hierarchy.

\section{A. Multi-Hamiltonian structure}

We consider the following vector Kaup-Newell spectral problem [2]

$$
\Psi_{x}=\left(\mathrm{i} \lambda^{-2} \sigma_{3}+\lambda^{-1} Q\right) \Psi \equiv U(\lambda) \Psi, \Psi=\left(\begin{array}{c}
\psi_{1} \\
\psi_{2}
\end{array}\right), \sigma_{3}=\left(\begin{array}{cc}
1 & 0 \\
0 & -I_{N}
\end{array}\right), Q=\left(\begin{array}{cc}
0 & \mathbf{r}^{\mathrm{T}} \\
\mathbf{q} & 0
\end{array}\right)
$$

where $\mathbf{q}=\left(q_{1}, q_{2}, \cdots, q_{N}\right)^{\mathrm{T}}, \mathbf{r}=\left(r_{1}, r_{2}, \cdots, r_{N}\right)^{\mathrm{T}}, I_{N}$ denotes a $N \times N$ identity matrix, $\lambda$ is the spectral parameter. To obtain the soliton hierarchy and its Hamiltonian structure, we consider the stationary zero-curvature equation

$$
W_{x}=[U, W], \quad W=\left(\begin{array}{cc}
a & \mathbf{c}^{\mathrm{T}} \\
\mathbf{b} & \mathbf{d}
\end{array}\right)
$$

where $\mathbf{b}$ and $\mathbf{c}$ are $N \times 1$ matrices, $\mathbf{d}$ is a $N \times N$ matrix. The equation (3) can be expanded as

$$
\begin{array}{ll}
a_{x}=\lambda^{-1}\left(\mathbf{r}^{\mathrm{T}} \mathbf{b}-\mathbf{c}^{\mathrm{T}} \mathbf{q}\right) & \mathbf{d}_{x}=\lambda^{-1}\left(\mathbf{q} \mathbf{c}^{\mathrm{T}}-\mathbf{b} \mathbf{r}^{\mathrm{T}}\right), \\
\mathbf{b}_{x}=-2 \mathrm{i} \lambda^{-2} \mathbf{b}+\lambda^{-1}(a \mathbf{q}-\mathbf{d} \mathbf{q}), & \mathbf{c}_{x}=2 \mathrm{i} \lambda^{-2} \mathbf{c}+\lambda^{-1}\left(\mathbf{d}^{\mathrm{T}} \mathbf{r}-a \mathbf{r}\right) .
\end{array}
$$

Firstly, we consider the positive hierarchy. To obtain the recursion operator for the positive hierarchy, we insert equations (5) into equations (4) to obtain

$$
\begin{aligned}
& a_{x}=\frac{\mathrm{i}}{2} \lambda\left(\mathbf{r}^{\mathrm{T}} \mathbf{b}_{x}+\mathbf{c}_{x}^{\mathrm{T}} \mathbf{q}\right), \\
& \mathbf{d}_{x}=-\frac{\mathrm{i} \lambda}{2}\left(\mathbf{q} \mathbf{c}_{x}^{\mathrm{T}}+\mathbf{b}_{x} \mathbf{r}^{\mathrm{T}}\right)+\frac{\mathrm{i}}{2}\left(\left(\mathbf{q} \mathbf{r}^{\mathrm{T}}\right) \mathbf{d}-\mathbf{d}\left(\mathbf{q} \mathbf{r}^{\mathrm{T}}\right)\right) .
\end{aligned}
$$

Then inserting equation (6) into equation (5) yields

$$
\begin{aligned}
& \mathrm{i} \lambda^{-2} \mathbf{b}=-\frac{1}{2} \mathbf{b}_{x}+\frac{1}{2} \lambda^{-1} a \mathbf{q}-\frac{1}{2} \lambda^{-1} \mathbf{d q}, \\
& \mathrm{i} \lambda^{-2} \mathbf{c}=\frac{1}{2} \mathbf{c}_{x}-\frac{1}{2} \lambda^{-1} \mathbf{d r}+\frac{1}{2} \lambda^{-1} a \mathbf{r} .
\end{aligned}
$$


Finally, supposing $W$ to be of the form

$$
\begin{aligned}
& a=\sum_{i=0}^{\infty} a_{i} \lambda^{2 i}, \mathbf{d}=\sum_{i=0}^{\infty} \mathbf{d}_{i} \lambda^{2 i} \\
& \mathbf{b}=\sum_{i=0}^{\infty} \mathbf{b}_{i} \lambda^{2 i+1}, \mathbf{c}=\sum_{i=0}^{\infty} \mathbf{c}_{i} \lambda^{2 i+1}
\end{aligned}
$$

we can obtain that

$$
\begin{aligned}
& a_{0}=2 \alpha_{0}, \mathbf{d}_{0}=-2 \alpha_{0} I_{N}, b_{0}=\mathbf{q} \alpha_{0}, \mathbf{c}_{0}=\mathbf{r} \alpha_{0}, \\
& a_{i+1, x}=\frac{\mathrm{i}}{2}\left(\mathbf{r}^{\mathrm{T}} \mathbf{b}_{i, x}+\mathbf{c}_{i, x}^{\mathrm{T}} \mathbf{q}\right), \mathrm{d}_{i+1, x}-\frac{\mathrm{i}}{2} \operatorname{ad}_{\mathbf{q r} \mathbf{r}^{\mathrm{T}}} \mathbf{d}_{i+1}=-\frac{\mathrm{i}}{2}\left(\mathbf{b}_{i, x} \mathbf{r}^{\mathrm{T}}+\mathbf{q} \mathbf{c}_{i, x}^{\mathrm{T}}\right), \\
& \mathrm{i} \mathbf{b}_{i+1}=-\frac{1}{2} \mathbf{b}_{i, x}+\frac{1}{2}\left(\mathbf{q} a_{i+1}-\mathbf{d}_{i+1} \mathbf{q}\right), \quad i \mathbf{c}_{i+1}=\frac{1}{2} \mathbf{c}_{i, x}-\frac{1}{2}\left(\mathbf{d}_{i+1}^{\mathrm{T}} \mathbf{r}-\mathbf{r} a_{i+1}\right),
\end{aligned}
$$

where $\alpha_{0}$ is a complex constant, $\operatorname{ad}_{\mathbf{q r}^{\mathrm{T}}}(\cdot)=\left[\mathbf{q} \mathbf{r}^{\mathrm{T}}, \cdot\right]$. The term $-\frac{\mathrm{i}}{2} \operatorname{ad}_{\mathbf{q r}} \mathbf{d}_{i+1}$ does not occur in the scalar equation since it is commutative automatically. Based on the above equations, one can obtain the recursion relation

$$
\left(\begin{array}{l}
\mathbf{b}_{i+1} \\
\mathbf{c}_{i+1}
\end{array}\right)=L_{1} \partial_{x}\left(\begin{array}{l}
\mathbf{b}_{i} \\
\mathbf{c}_{i}
\end{array}\right)
$$

where

$$
L_{1}=\frac{\mathrm{i}}{2}\left(\begin{array}{cc}
I_{N} & 0 \\
0 & -I_{N}
\end{array}\right)+\frac{1}{4}\left(\begin{array}{cc}
\mathbf{q} \partial_{x}^{-1} \mathbf{r}^{\mathrm{T}}+A & \mathbf{q} \partial_{x}^{-1} \mathbf{q}^{\mathrm{T}}+C \\
\mathbf{r} \partial_{x}^{-1} \mathbf{r}^{\mathrm{T}}+D & \mathbf{r} \partial_{x}^{-1} \mathbf{q}^{\mathrm{T}}+B
\end{array}\right)
$$

and

$$
\begin{aligned}
& A=\left[\left(\partial_{x}-\frac{\mathrm{i}}{2} \operatorname{ad}_{\mathbf{q} \mathbf{r}^{\mathrm{T}}}\right)^{-1}\left((\cdot) \mathbf{r}^{\mathrm{T}}\right)\right] \mathbf{q}, \quad B=\left[\left(\partial_{x}+\frac{\mathrm{i}}{2} \operatorname{ad}_{\mathbf{r} \mathbf{q}^{\mathrm{T}}}\right)^{-1}\left((\cdot) \mathbf{q}^{\mathrm{T}}\right)\right] \mathbf{r} \\
& C=\left[\left(\partial_{x}-\frac{\mathrm{i}}{2} \operatorname{ad}_{\mathbf{q} \mathbf{r}^{\mathrm{T}}}\right)^{-1}\left(\mathbf{q}(\cdot)^{\mathrm{T}}\right)\right] \mathbf{q}, \quad D=\left[\left(\partial_{x}+\frac{\mathrm{i}}{2} \operatorname{ad}_{\mathbf{r q}^{\mathrm{T}}}\right)^{-1}\left(\mathbf{r}(\cdot)^{\mathrm{T}}\right)\right] \mathbf{r} .
\end{aligned}
$$

\section{Proposition 1}

$$
A^{*}=-B, C^{*}=-C, D^{*}=-D
$$

Proof: By direct calculation, we have

$$
\begin{aligned}
& \int \mathbf{c}^{\mathrm{T}}\left[\left(\partial_{x}-\frac{\mathrm{i}}{2} \operatorname{ad}_{\mathbf{q r}^{\mathrm{T}}}\right)\left(\mathbf{b} \mathbf{r}^{\mathrm{T}}\right)\right] \mathbf{q} \mathrm{d} x \\
= & \int \mathbf{c}^{\mathrm{T}}\left[\left(\mathbf{b r}^{\mathrm{T}}\right)_{x}-\frac{\mathrm{i}}{2} \operatorname{ad}_{\mathbf{q r}} \mathbf{r}^{\mathrm{T}}\left(\mathbf{b r} \mathbf{T}^{\mathrm{T}}\right)\right] \mathbf{q} \mathrm{d} x \\
= & \int\left\{-\mathbf{c}_{x}^{\mathrm{T}}\left(\mathbf{b} \mathbf{r}^{\mathrm{T}}\right) \mathbf{q}-\mathbf{c}^{\mathrm{T}}\left(\mathbf{b} \mathbf{r}^{\mathrm{T}}\right) \mathbf{q}_{x}-\frac{\mathrm{i}}{2} \mathbf{c}^{\mathrm{T}}\left[\operatorname{ad}_{\mathbf{q r}^{\mathrm{T}}}\left(\mathbf{b} \mathbf{r}^{\mathrm{T}}\right)\right] \mathbf{q}\right\} \mathrm{d} x \\
= & \int \mathbf{b}^{\mathrm{T}}\left[\left(-\partial_{x}-\frac{\mathrm{i}}{2} \operatorname{ad}_{\mathbf{r} \mathbf{q}^{\mathrm{T}}}\right)\left(\mathbf{c q}^{\mathrm{T}}\right)\right] \mathbf{r} \mathrm{d} x .
\end{aligned}
$$

Thus, we can obtain that $A^{*}=-B$ formally. Other two cases can be proved in a similar way.

From the trace identity, one has

$$
\frac{\delta}{\delta \omega} \int \operatorname{tr}\left(W \frac{\partial U}{\partial \lambda}\right) \mathrm{d} x=\lambda^{-\gamma} \frac{\partial}{\partial \lambda}\left[\lambda^{\gamma} \operatorname{tr}\left(W \frac{\partial U}{\partial \omega}\right)\right], \quad \omega=\left(\begin{array}{l}
\mathbf{q} \\
\mathbf{r}
\end{array}\right) .
$$

Since

$$
\begin{aligned}
\operatorname{tr}\left(W \frac{\partial U}{\partial \lambda}\right) & =-2 \mathrm{i} \lambda^{-3}[a-\operatorname{tr}(\mathbf{d})]-\lambda^{-2}\left(\mathbf{c}^{\mathrm{T}} \mathbf{q}+\mathbf{r}^{\mathrm{T}} \mathbf{b}\right) \\
& =-2 \mathrm{i}\left[a_{0}-\operatorname{tr}\left(\mathbf{d}_{0}\right)\right]-\sum_{i=0}^{\infty}\left[2 \mathrm{i}\left[a_{i+1}-\operatorname{tr}\left(\mathbf{d}_{i+1}\right)\right]+\left(\mathbf{c}_{i}^{\mathrm{T}} \mathbf{q}+\mathbf{r}^{\mathrm{T}} \mathbf{b}_{i}\right)\right] \lambda^{2 i-1}
\end{aligned}
$$


and

$$
\operatorname{tr}\left(W \frac{\partial U}{\partial \omega}\right)=\lambda^{-1}\left(\begin{array}{l}
\mathbf{c} \\
\mathbf{b}
\end{array}\right)
$$

it then follows that

$$
\left(\begin{array}{l}
\mathbf{c}_{0} \\
\mathbf{b}_{0}
\end{array}\right)=\frac{\delta}{\delta \omega} H_{0}^{+}, H_{0}^{+}=\alpha_{0} \int \mathbf{q}^{\mathrm{T}} \cdot \mathbf{r d} x
$$

and

$$
\left(\begin{array}{c}
\mathbf{c}_{i} \\
\mathbf{b}_{i}
\end{array}\right)=\frac{\delta}{\delta \omega} H_{i}^{+}, H_{i}^{+}=-\frac{1}{2 i} \int\left[2 \mathrm{i}\left(a_{i+1}-\operatorname{tr}\left(\mathbf{d}_{i+1}\right)\right)+\left(\mathbf{c}_{i}^{\mathrm{T}} \mathbf{q}+\mathbf{r}^{\mathrm{T}} \mathbf{b}_{i}\right)\right] \mathrm{d} x, i \geq 1 .
$$

Finally, setting $V(\lambda)=\left(\lambda^{-2 k} W\right)_{-}$, we obtain the multi-Hamiltonian hierarchy

$$
\omega_{t}=J \frac{\delta}{\delta \omega} H_{n}^{+}=J L^{n} \frac{\delta}{\delta \omega} H_{0}^{+},
$$

from the zero curvature condition $U_{t}-V_{x}+[U, V]=0$, where

$$
J=\left(\begin{array}{ll}
0 & \partial \\
\partial & 0
\end{array}\right), L=\left(\begin{array}{cc}
0 & I_{N} \\
I_{N} & 0
\end{array}\right) L_{1}\left(\begin{array}{cc}
0 & I_{N} \\
I_{N} & 0
\end{array}\right) \partial
$$

It is readily to see that the operators $J$ and $J L^{k}$ are skew symmetrical.

On the other hand, by assuming

$$
\begin{aligned}
& a=\sum_{i=0}^{\infty} a_{i} \lambda^{-2 i}, \mathbf{d}=\sum_{i=0}^{\infty} \mathbf{d}_{i} \lambda^{-2 i} \\
& \mathbf{b}=\sum_{i=0}^{\infty} \mathbf{b}_{i} \lambda^{-2 i-1}, \mathbf{c}=\sum_{i=0}^{\infty} \mathbf{c}_{i} \lambda^{-2 i-1}
\end{aligned}
$$

one obtains the recursion relation

$$
\begin{aligned}
& \left(\begin{array}{l}
\mathbf{b}_{i} \\
\mathbf{c}_{i}
\end{array}\right)_{x}=L_{2}\left(\begin{array}{l}
\mathbf{b}_{i-1} \\
\mathbf{c}_{i-1}
\end{array}\right), i \geq 1,\left(\begin{array}{l}
\mathbf{b}_{0} \\
\mathbf{c}_{0}
\end{array}\right)_{x}=\alpha_{0}\left(\begin{array}{l}
\mathbf{q} \\
\mathbf{r}
\end{array}\right), \\
& a_{0}=\frac{1}{2} \alpha_{0}, \mathbf{d}_{0}=-\frac{1}{2} \alpha_{0} I_{N}, a_{i}=\partial_{x}^{-1}\left(\mathbf{r}^{\mathrm{T}} \mathbf{b}_{i-1}-\mathbf{c}_{i-1}^{\mathrm{T}} \mathbf{q}\right), \mathbf{d}_{i}=\partial_{x}^{-1}\left(\mathbf{q c}_{i-1}^{\mathrm{T}}-\mathbf{b}_{i-1} \mathbf{r}^{\mathrm{T}}\right) \text { and } \\
& L_{2}=-2 \mathrm{i}\left(\begin{array}{cc}
I_{N} & 0 \\
0 & -I_{N}
\end{array}\right)+\left(\begin{array}{cc}
\mathbf{q} \partial_{x}^{-1} \mathbf{r}^{\mathrm{T}}+\left(\sum_{k=1}^{N} q_{k} \partial_{x}^{-1} r_{k}\right) I_{N} & -\mathbf{q} \partial_{x}^{-1} \mathbf{q}^{\mathrm{T}}-\left(\mathbf{q} \partial_{x}^{-1} \mathbf{q}^{\mathrm{T}}\right)^{\mathrm{T}} \\
-\mathbf{r} \partial_{x}^{-1} \mathbf{r}^{\mathrm{T}}-\left(\mathbf{r} \partial_{x}^{-1} \mathbf{r}^{\mathrm{T}}\right)^{\mathrm{T}} & \mathbf{r} \partial_{x}^{-1} \mathbf{q}^{\mathrm{T}}+\left(\sum_{k=1}^{N} r_{k} \partial_{x}^{-1} q_{k}\right) I_{N}
\end{array}\right) .
\end{aligned}
$$

Through the trace identity (9), we can obtain that

$$
\left(\begin{array}{c}
\mathbf{c}_{i} \\
\mathbf{b}_{i}
\end{array}\right)=\frac{\delta}{\delta \omega} H_{i}^{-}, H_{i}^{-}=\int \frac{1}{2(i+1)}\left[2 \mathrm{i}\left(a_{i}-\operatorname{tr}\left(\mathbf{d}_{i}\right)\right)+\mathbf{c}_{i}^{\mathrm{T}} \mathbf{q}+\mathbf{r}^{\mathrm{T}} \mathbf{b}_{i}\right] \mathrm{d} x .
$$

Taking $V(\lambda)=\left(\lambda^{2 n} W\right)_{+}$, we can obtain the negative hierarchy

$$
\omega_{t}=-J \frac{\delta}{\delta \omega} H_{n}=-J K^{n} \frac{\delta}{\delta \omega} H_{0}
$$

where

$$
K=\partial_{x}^{-1}\left(\begin{array}{cc}
0 & I_{N} \\
I_{N} & 0
\end{array}\right) L_{2}\left(\begin{array}{cc}
0 & I_{N} \\
I_{N} & 0
\end{array}\right)
$$

It can be readily verified that the operator $J K^{i}$ is skew symmetric. Consequently, both the positive and negative flows of the multi-component Kaup-Newell hierarchy are constructed by Tu scheme. 


\section{B. Conservation laws}

We consider the multi-component Kaup-Newell spectral problem

$$
\left(\begin{array}{l}
\psi_{1} \\
\psi_{2}
\end{array}\right)_{x}=\left(\begin{array}{cc}
\mathrm{i} \lambda^{-2} & \lambda^{-1} \mathbf{v}_{x}^{T} \\
\lambda^{-1} \mathbf{u}_{x} & -\mathrm{i} \lambda^{-2}
\end{array}\right)\left(\begin{array}{l}
\psi_{1} \\
\psi_{2}
\end{array}\right)
$$

and associated evolution equation

$$
\left(\begin{array}{l}
\psi_{1} \\
\psi_{2}
\end{array}\right)_{t}=\mathrm{i}\left(\begin{array}{cc}
\frac{1}{4} \lambda^{2}+\frac{1}{2} \mathbf{v}^{\mathrm{T}} \mathbf{u} & -\frac{\lambda}{2} \mathbf{v}^{\mathrm{T}} \\
\frac{\lambda}{2} \mathbf{u} & -\lambda^{2} I_{N}-\frac{1}{2} \mathbf{u} \mathbf{v}^{\mathrm{T}}
\end{array}\right)\left(\begin{array}{l}
\psi_{1} \\
\psi_{2}
\end{array}\right)
$$

It follows that

$$
\mathbf{v}_{x}^{\mathrm{T}}\left(\frac{\psi_{2}}{\psi_{1}}\right)_{x}=-2 \mathrm{i} \lambda^{-2} \mathbf{v}_{x}^{\mathrm{T}} \frac{\psi_{2}}{\psi_{1}}+\lambda^{-1} \mathbf{v}_{x}^{\mathrm{T}} \mathbf{u}_{x}-\lambda^{-1}\left(\frac{\mathbf{v}_{x}^{\mathrm{T}} \psi_{2}}{\psi_{1}}\right)^{2}
$$

and

$$
\mathbf{v}^{\mathrm{T}}\left(\frac{\psi_{2}}{\psi_{1}}\right)_{t}=-\frac{\mathrm{i}}{2} \lambda^{2} \mathbf{v}^{\mathrm{T}} \frac{\psi_{2}}{\psi_{1}}+\frac{\mathrm{i}}{2} \lambda \mathbf{v}^{\mathrm{T}} \mathbf{u}-\mathrm{i}\left(\mathbf{v}^{\mathrm{T}} \mathrm{u}\right) \mathbf{v}^{\mathrm{T}} \frac{\psi_{2}}{\psi_{1}}+\frac{\mathrm{i} \lambda}{2}\left(\frac{\mathbf{v}^{\mathrm{T}} \psi_{2}}{\psi_{1}}\right)^{2} .
$$

To find the conservation laws of negative orders, we substitute an expansion

$$
\frac{\psi_{2}}{\psi_{1}}=\sum_{i=1}^{\infty} \mathbf{P}_{i} \lambda^{2 i-1}
$$

into equation (12) and obtain

$$
\begin{aligned}
\mathbf{P}_{0} & =\mathbf{u}, \quad \mathbf{P}_{1}=-\frac{\mathrm{i}}{2} \mathbf{u}_{x} \\
\mathbf{P}_{i+1} & =\frac{\mathrm{i}}{2}\left[\mathbf{P}_{i, x}+\sum_{j=1}^{i} \mathbf{P}_{j} \mathbf{v}_{x}^{\mathrm{T}} \mathbf{P}_{i+1-j}\right] .
\end{aligned}
$$

Then the conservation laws follow

$$
\left(\ln \psi_{1}\right)_{x t}=\lambda^{-1}\left[\mathbf{v}_{x}^{\mathrm{T}} \frac{\psi_{2}}{\psi_{1}}\right]_{t}=\mathrm{i}\left[\frac{1}{2}\left(\mathbf{v}^{\mathrm{T}} \mathrm{u}\right)_{x}-\frac{\lambda}{2}\left(\mathbf{v}^{\mathrm{T}} \frac{\psi_{2}}{\psi_{1}}\right)_{x}\right],
$$

i.e.

$$
\mathrm{i}\left[\mathbf{v}_{x}^{\mathrm{T}} \mathbf{P}_{i}\right]_{t}=\frac{1}{2}\left(\mathbf{v}^{\mathrm{T}} \mathbf{P}_{i-1}\right)_{x}, \quad i=1,2, \cdots
$$

These conservation laws are all local, among which the first two are listed below

$$
\begin{aligned}
{\left[\mathbf{v}_{x}^{\mathrm{T}} \mathbf{u}_{x}\right]_{t} } & =\frac{\mathrm{i}}{2}\left(\mathbf{v}^{\mathrm{T}} \mathbf{u}\right)_{x}, \\
{\left[\frac{\mathrm{i}}{4} \mathbf{v}^{\mathrm{T}} \mathbf{u}_{x x}+\frac{1}{8}\left(\mathbf{v}^{\mathrm{T}} \mathbf{u}_{x}\right)^{2}\right]_{t} } & =\frac{1}{4}\left(\mathbf{v}^{\mathrm{T}} \mathbf{u}_{x}\right)_{x} .
\end{aligned}
$$

On the other hand, substituting the following expansion

$$
\frac{\psi_{2}}{\psi_{1}}=\sum_{i=1}^{\infty} \mathbf{C}_{i} \lambda^{-2 i+1}
$$

into equation (13) and equating the coefficients of $\lambda^{-2 i+1}$, one obtains

$$
\begin{aligned}
\lambda: & \mathbf{C}_{1}=\mathbf{u}, \\
\lambda^{-2 i+1}: & \mathbf{v}^{\mathrm{T}} \mathbf{C}_{i+1}=2 \mathrm{i} \mathbf{v}^{\mathrm{T}} \mathbf{C}_{i, t}-2\left(\mathbf{v}^{\mathrm{T}} \mathbf{u}\right)\left(\mathbf{v}^{\mathrm{T}} \mathbf{C}_{i}\right)+\sum_{j=1}^{i}\left(\mathbf{v}^{\mathrm{T}} \mathbf{C}_{j}\right)\left(\mathbf{v}^{\mathrm{T}} \mathbf{C}_{i+1-j}\right),
\end{aligned}
$$


which leads to the conservation laws

$$
\left(\ln \psi_{1}\right)_{x t}=\lambda^{-1}\left[\mathbf{v}_{x}^{\mathrm{T}} \frac{\psi_{2}}{\psi_{1}}\right]_{t}=\mathrm{i}\left[\frac{1}{2}\left(\mathbf{v}^{\mathrm{T}} \mathbf{u}\right)_{x}-\frac{\lambda}{2}\left(\mathbf{v}^{\mathrm{T}} \frac{\psi_{2}}{\psi_{1}}\right)_{x}\right]
$$

i.e.

$$
\mathrm{i}\left[\mathbf{v}_{x}^{\mathrm{T}} \mathbf{C}_{i}\right]_{t}=\left[\frac{1}{2} \mathbf{v}^{\mathrm{T}} \mathbf{C}_{i+1}\right]_{x}, \quad i=1,2, \cdots .
$$

The first conversation law is

$$
\mathrm{i}\left[\mathbf{v}_{x}^{\mathrm{T}} \mathbf{u}\right]_{t}=\left[\mathrm{iv}^{\mathrm{T}} \mathbf{u}_{t}-\frac{1}{2}\left(\mathbf{v}^{\mathrm{T}} \mathbf{u}\right)^{2}\right]_{x} .
$$

In contrast to the conservation laws of negative orders, other conservation laws except the first one of positive orders are nonlocal.

\section{SPECTRAL PROBLEM AND DARBOUX TRANSFORMATION}

The coupled FL equation (11) considered in the present paper admits the following Lax pair

$$
\begin{array}{ll}
\Phi_{x}=U(x, t ; \lambda) \Phi, \quad U(x, t ; \lambda)=\mathrm{i} \lambda^{-2} \sigma_{3}+\lambda^{-1} Q_{x}, \\
\Phi_{t}=V(x, t ; \lambda) \Phi, \quad V(x, t ; \lambda)=\mathrm{i}\left(\frac{1}{4} \lambda^{2} \sigma_{3}+\frac{1}{2} \sigma_{3}\left(Q^{2}-\lambda Q\right)\right),
\end{array}
$$

where

$$
\sigma_{3}=\operatorname{diag}(1,-1,-1), \quad Q=\left(\begin{array}{ccc}
0 & u_{1}^{*} & \sigma u_{2}^{*} \\
u_{1} & 0 & 0 \\
u_{2} & 0 & 0
\end{array}\right), \quad \sigma= \pm 1 .
$$

So the solutions to equation (1) can be solved from its Lax pair (16). To the end, we consider the spectral problem (16)

$$
-\mathrm{i} \sigma_{3}\left[\partial_{x}-\lambda^{-1} Q_{x}\right] \Phi=\lambda^{-2} \Phi,
$$

which is an energy-dependent spectral problem, here $Q \in L_{l o c}^{1}(\mathbb{R})$. The involution relation for the system (16) can be concluded by the following Lemma:

Lemma 1 (a) The matrices $U, V$ are variant under the involution $\tau_{1}: A(\lambda) \mapsto \sigma_{3} A(-\lambda) \sigma_{3}$ and $\tau_{2}: A(\lambda) \mapsto$ $-J\left[A\left(\lambda^{*}\right)\right]^{\dagger} J$, where $J=\operatorname{diag}(1,-1,-\sigma)$. (b) If the function $\Phi(x, t ; \lambda)$ satisfies the system (16) with initial data $\Phi(0,0 ; \lambda)=I_{3 \times 3}$, then $\Phi(x, t ;-\lambda)=\sigma_{3} \Phi(x, t ; \lambda) \sigma_{3}$, and $J[\Phi(x, t ; \lambda)]^{\dagger} J=\left[\Phi\left(x, t ; \lambda^{*}\right)\right]^{-1}$.

Proof: The claim in (a) can be proved by direct calculation, which is omitted here.

b) Since $\Phi(x, t ; \lambda)$ satisfies the system (16) with initial data $\Phi(0,0 ; \lambda)=I_{3 \times 3}$, and $U, V$ are invariant under the involution $\tau_{1}$, we have

$$
\begin{aligned}
\frac{\partial}{\partial x} \Phi(x, t ;-\lambda) & =U(x, t ;-\lambda) \Phi(x, t ;-\lambda), \\
\frac{\partial}{\partial t} \Phi(x, t ;-\lambda) & =V(x, t ;-\lambda) \Phi(x, t ;-\lambda),
\end{aligned}
$$

and

$$
\begin{aligned}
\frac{\partial}{\partial x}\left[\sigma_{3} \Phi(x, t ; \lambda) \sigma_{3}\right] & =U(x, t ;-\lambda)\left[\sigma_{3} \Phi(x, t ; \lambda) \sigma_{3}\right], \\
\frac{\partial}{\partial t}\left[\sigma_{3} \Phi(x, t ; \lambda) \sigma_{3}\right] & =V(x, t ;-\lambda)\left[\sigma_{3} \Phi(x, t ; \lambda) \sigma_{3}\right] .
\end{aligned}
$$


Based on the existence and uniqueness of ordinary differential equations (ODEs), we have $\Phi(x, t ;-\lambda)=\sigma_{3} \Phi(x, t ; \lambda) \sigma_{3}$.

Similarly, since $\Phi(x, t ; \lambda)$ satisfies the system (16) with initial data $\Phi(0,0 ; \lambda)=I_{3 \times 3}$, and $U, V$ are invariant under the involution $\tau_{2}$, one has

$$
\begin{aligned}
& -\left(J[\Phi(x, t ; \lambda)]^{\dagger} J\right)_{x}=\left(J[\Phi(x, t ; \lambda)]^{\dagger} J\right) U\left(x, t ; \lambda^{*}\right), \\
& -\left(J[\Phi(x, t ; \lambda)]^{\dagger} J\right)_{t}=\left(J[\Phi(x, t ; \lambda)]^{\dagger} J\right) V\left(x, t ; \lambda^{*}\right)
\end{aligned}
$$

and

$$
\begin{aligned}
& -\left([\Phi(x, t ; \lambda)]^{-1}\right)_{x}=\left([\Phi(x, t ; \lambda)]^{-1}\right) U\left(x, t ; \lambda^{*}\right), \\
& -\left([\Phi(x, t ; \lambda)]^{-1}\right)_{t}=\left([\Phi(x, t ; \lambda)]^{-1}\right) V\left(x, t ; \lambda^{*}\right),
\end{aligned}
$$

we then have $J[\Phi(x, t ; \lambda)]^{\dagger} J=\left[\Phi\left(x, t ; \lambda^{*}\right)\right]^{-1}$.

Therefore, the Darboux matrix $T(\lambda)$ converts the system (16) into a new system of the form

$$
\begin{array}{ll}
\Phi[1]_{x}=U[1](x, t ; \lambda) \Phi[1], & U[1](x, t ; \lambda)=\mathrm{i} \lambda^{-2} \sigma_{3}+\lambda^{-1} Q[1]_{x}, \\
\Phi[1]_{t}=V[1](x, t ; \lambda) \Phi[1], & V[1](x, t ; \lambda)=\mathrm{i}\left(\frac{1}{4} \lambda^{2} \sigma_{3}+\frac{1}{2} \sigma_{3}\left(Q[1]^{2}-\lambda Q[1]\right)\right) .
\end{array}
$$

where

$$
\begin{aligned}
& U[1]=T_{x} T^{-1}+T U T^{-1}, \\
& V[1]=T_{t} T^{-1}+T V T^{-1} .
\end{aligned}
$$

Lemma 2 Assume that $T(\lambda)$ satisfies $T(\lambda)=\sigma_{3} T(-\lambda) \sigma_{3}$ and $[T(\lambda)]^{-1}=J\left[T\left(\lambda^{*}\right)\right]^{\dagger} J$, if the matrices $U$, $V$ are invariant under the involution $\tau_{1}$ and $\tau_{2}$, then the new potential functions keep invariant under the involution $\tau_{1}$ and $\tau_{2}$

Proof: From the relation (19) and $T(\lambda)=\sigma_{3} T(-\lambda) \sigma_{3}$, we have

$$
\begin{aligned}
& \sigma_{3} U[1](-\lambda) \sigma_{3}=\sigma_{3} T(-\lambda)_{x}[T(-\lambda)]^{-1} \sigma_{3}+\sigma_{3} T(-\lambda) U(-\lambda)[T(-\lambda)]^{-1} \sigma_{3}=U[1](\lambda), \\
& \sigma_{3} V[1](-\lambda) \sigma_{3}=\sigma_{3} T(-\lambda)_{t}[T(-\lambda)]^{-1} \sigma_{3}+\sigma_{3} T(-\lambda) V(-\lambda)[T(-\lambda)]^{-1} \sigma_{3}=V[1](\lambda) .
\end{aligned}
$$

On the other hand, through the relation (19) and $[T(\lambda)]^{-1}=J\left[T\left(\lambda^{*}\right)\right]^{\dagger} J$, one obtains

$$
\begin{aligned}
J\left[U[1]\left(\lambda^{*}\right)\right]^{\dagger} J & =\sigma_{3} T(\lambda)_{x}[T(\lambda)]^{-1} \sigma_{3}+\sigma_{3} T(\lambda) U(\lambda)[T(\lambda)]^{-1} \sigma_{3}=U[1](\lambda), \\
\sigma_{3} V[1](\lambda) \sigma_{3} & =\sigma_{3} T(-\lambda)_{t}[T(-\lambda)]^{-1} \sigma_{3}+\sigma_{3} T(-\lambda) V(-\lambda)[T(-\lambda)]^{-1} \sigma_{3}=V[1](\lambda) .
\end{aligned}
$$

Due to the relation $T(\lambda)=\sigma_{3} T(-\lambda) \sigma_{3}$, the Darboux matrix can be constructed through the loop group method

$$
T(\lambda)=I+\frac{A_{1}}{\lambda-\lambda_{1}^{*}}-\frac{\sigma_{3} A_{1} \sigma_{3}}{\lambda+\lambda_{1}^{*}} .
$$

Based on the Lemma 2. the inverse of Darboux matrix $T(\lambda)$ may be chosen as

$$
T(\lambda)^{-1}=J\left[T\left(\lambda^{*}\right)\right]^{\dagger} J=I+\frac{J A_{1}^{\dagger} J}{\lambda-\lambda_{1}}-\frac{\sigma_{3} J A_{1}^{\dagger} J \sigma_{3}}{\lambda+\lambda_{1}} .
$$

The $L^{2}(\mathbb{R})$ eigenfunction can be constructed from the Darboux matrix. Usually, we can construct two wave vector functions which satisfy $\phi_{ \pm}\left(\lambda_{1}\right) \rightarrow 0$ as $x \rightarrow \pm \infty$ with exponential decay, $\lambda_{1} \in \mathbb{C} /\{\mathbb{R} \cup \mathrm{i}\}$. The Darboux matrix satisfies the following proposition

$$
T\left(\lambda_{1}\right)\left|y_{1}\right\rangle=0,\left|y_{1}\right\rangle=\phi_{1,+}+\gamma \phi_{1,-},
$$

where $\operatorname{rank}\left(A_{1}\right)$ is either 1 or 2 . However, since the order for this spectral problem is three, we can assume that $A_{1}=\left|x_{1}\right\rangle\left\langle z_{1}\right| J$, where $\left\langle z_{1}|=| z_{1}\right\rangle^{\dagger}$. On the other hand, since $T(\lambda)[T(\lambda)]^{-1}=I$, one can obtain that $\operatorname{Res}_{\lambda=\lambda_{1}}\left(T(\lambda)[T(\lambda)]^{-1}\right)=0$. It follows that

$$
\left[I+\frac{A_{1}}{\lambda_{1}-\lambda_{1}^{*}}-\frac{\sigma_{3} A_{1} \sigma_{3}}{\lambda_{1}+\lambda_{1}^{*}}\right]\left|z_{1}\right\rangle=0
$$


Together with equation (22), one arrives at $\left|z_{1}\right\rangle=c_{1}\left|y_{1}\right\rangle$. For the sake of convenience, by setting $c_{1}=1$, equation (23) is rewritten as

$$
\left|y_{1}\right\rangle+\frac{\left\langle y_{1}|J| y_{1}\right\rangle}{\lambda_{1}-\lambda_{1}^{*}}\left|x_{1}\right\rangle-\frac{\left\langle y_{1}\left|J \sigma_{3}\right| y_{1}\right\rangle}{\lambda_{1}+\lambda_{1}^{*}} \sigma_{3}\left|x_{1}\right\rangle=0
$$

Denoting $\left|y_{1}\right\rangle=\left(\varphi_{1}, \psi_{1}, \chi_{1}\right)^{\mathrm{T}}$, one can solve

$$
\left|x_{1}\right\rangle=\left(\begin{array}{ccc}
\alpha^{-1} & 0 & 0 \\
0 & \beta^{-1} & 0 \\
0 & 0 & \beta^{-1}
\end{array}\right)\left|y_{1}\right\rangle
$$

where

$$
\alpha=\frac{2\left[\lambda_{1}^{*}\left|\varphi_{1}\right|^{2}-\lambda_{1}\left(\left|\psi_{1}\right|^{2}+\sigma\left|\chi_{1}\right|^{2}\right)\right]}{\lambda_{1}^{* 2}-\lambda_{1}^{2}}, \beta=\frac{2\left[\lambda_{1}\left|\varphi_{1}\right|^{2}-\lambda_{1}^{*}\left(\left|\psi_{1}\right|^{2}+\sigma\left|\chi_{1}\right|^{2}\right)\right]}{\lambda_{1}^{* 2}-\lambda_{1}^{2}} .
$$

Consequently, we obtain the transformation from potential function $Q$ to $Q[1]$

$$
Q[1]=Q+\left(\left|x_{1}\right\rangle\left\langle y_{1}\left|J-\sigma_{3}\right| x_{1}\right\rangle\left\langle y_{1}\right| J \sigma_{3}\right),
$$

or explicitly

$$
\begin{aligned}
& u_{1}[1]=u_{1}+\frac{2}{\beta} \psi_{1} \varphi_{1}^{*}, \\
& u_{2}[1]=u_{2}+\frac{2}{\beta} \chi_{1} \varphi_{1}^{*} .
\end{aligned}
$$

Generally, we could derive the following $N$-fold Darboux transformation

$$
T_{N}=I+\sum_{i=1}^{N}\left[\frac{A_{i}}{\lambda-\lambda_{i}^{*}}-\frac{\sigma_{3} A_{i} \sigma_{3}}{\lambda+\lambda_{i}^{*}}\right]
$$

where $A_{i}=\left|x_{i}\right\rangle\left\langle y_{i}\right| J$

$$
\begin{aligned}
& {\left[\left|x_{1,1}\right\rangle,\left|x_{2,1}\right\rangle, \cdots,\left|x_{N, 1}\right\rangle\right]=\left[\left|y_{1,1}\right\rangle,\left|y_{2,1}\right\rangle, \cdots,\left|y_{N, 1}\right\rangle\right] B^{-1}, B=\left(b_{i j}\right)_{N \times N},} \\
& {\left[\left|x_{1, k}\right\rangle,\left|x_{2, k}\right\rangle, \cdots,\left|x_{N, k}\right\rangle\right]=\left[\left|y_{1, k}\right\rangle,\left|y_{2, k}\right\rangle, \cdots,\left|y_{N, k}\right\rangle\right] M^{-1}, M=\left(m_{i j}\right)_{N \times N}, \quad k=2,3,}
\end{aligned}
$$

and

$$
b_{i j}=\frac{\left\langle y_{i}|J| y_{j}\right\rangle}{\lambda_{i}^{*}-\lambda_{j}}+\frac{\left\langle y_{i}\left|J \sigma_{3}\right| y_{j}\right\rangle}{\lambda_{i}^{*}+\lambda_{j}}, m_{i j}=\frac{\left\langle y_{i}|J| y_{j}\right\rangle}{\lambda_{i}^{*}-\lambda_{j}}-\frac{\left\langle y_{i}\left|J \sigma_{3}\right| y_{j}\right\rangle}{\lambda_{i}^{*}+\lambda_{j}} .
$$

The transformation between old and new potential functions is

$$
Q[N]=Q+\sum_{i=1}^{N}\left(A_{i}-\sigma_{3} A_{i} \sigma_{3}\right) .
$$

Moreover, we have

$$
\left(\begin{array}{l}
u_{1}[N] \\
u_{2}[N]
\end{array}\right)=\left(\begin{array}{l}
u_{1} \\
u_{2}
\end{array}\right)+2 Y_{2} M^{-1} Y_{1}^{\dagger}
$$

where

$$
Y_{1}=\left(\begin{array}{llll}
\varphi_{1} & \varphi_{2} & \cdots & \varphi_{N}
\end{array}\right), Y_{2}=\left(\begin{array}{llll}
\phi_{1} & \phi_{2} & \cdots & \phi_{N} \\
\chi_{1} & \chi_{2} & \cdots & \chi_{N}
\end{array}\right)
$$




\section{BRIGHT SOLITON SOLUTION WITH VANISHING BOUNDARY CONDITION}

\section{A. Single bright soliton solution}

Inserting the zero seed solution into Lax pair (16) and introducing $z=1 / \lambda^{2}$, the fundamental solution to the system (16) is $\Phi_{1}(\lambda)=\exp \left[\mathrm{i}\left(z x+\frac{1}{4 z} t\right) \sigma_{3}\right]$. Based on the formula (26), one obtains the single soliton solution with vanishing boundary condition

$$
u_{s}[1]=\frac{z_{1}-z_{1}^{*}}{\left|z_{1}\right|^{2}} \frac{c_{s} \mathrm{e}^{*}}{\lambda_{1} \mathrm{e}^{\varpi_{1}+\varpi_{1}^{*}-\lambda_{1}^{*}\left(\left|c_{1}\right|^{2}+\sigma\left|c_{2}\right|^{2}\right)} .}
$$

Let $\lambda_{1}=\lambda_{1 R}+\mathrm{i} \lambda_{1 I}$ and $\varpi_{1}=\varpi_{1 R}+\mathrm{i} \varpi_{1 I}=\mathrm{i}\left(2 z_{1} x+\frac{1}{2 z_{1}} t\right)+\delta_{1}$, the single soliton solution can be represented as

$$
u_{s}[1]=\frac{-2 \lambda_{1 R} \lambda_{1 I} c_{s} \mathrm{i}^{-\mathrm{i} \varpi_{1 I}}}{r_{1}\left[\lambda_{1 R} \sinh \left(\varpi_{1 R}-\ln \left(r_{1}\right)\right)+\mathrm{i} \lambda_{1 I} \cosh \left(\varpi_{1 R}-\ln \left(r_{1}\right)\right)\right]}
$$

for $c_{1}^{2}+\sigma c_{2}^{2}>0$ and

$$
u_{s}[1]=\frac{-2 \lambda_{1 R} \lambda_{1 I} c_{s} \mathrm{ie}^{-\mathrm{i} \varpi_{1 I}}}{r_{1}\left[\lambda_{1 R} \cosh \left(\varpi_{1 R}-\ln \left(r_{1}\right)\right)+\mathrm{i} \lambda_{1 I} \sinh \left(\varpi_{1 R}-\ln \left(r_{1}\right)\right)\right]},
$$

for $c_{1}^{2}+\sigma c_{2}^{2}<0$. Here $r_{1}=\left(\left|c_{1}^{2}+\sigma c_{2}^{2}\right|\right)^{1 / 2}$ and

$$
\varpi_{1 R}=\lambda_{1 R} \lambda_{1 I}\left[\frac{4 x}{\left(\lambda_{1 R}^{2}+\lambda_{1 I}^{2}\right)^{2}}-t\right]+\delta_{1 R}, \varpi_{1 I}=\frac{\left(\lambda_{1 R}^{2}-\lambda_{1 I}^{2}\right)}{2}\left[\frac{4 x}{\left(\lambda_{1 R}^{2}+\lambda_{1 I}^{2}\right)^{2}}+t\right]+\delta_{1 I},
$$

where $\delta_{1 R}, \delta_{1 I} \in \mathbb{R}$. Basically, the soliton solution is the bright soliton solution of the bell shape. If $c_{1}^{2}+\sigma c_{2}^{2}>0$, the peak value for $\left|u_{s}[1]\right|^{2}$ is $4 \lambda_{1 R}^{2} c_{s}^{2} / r_{1}^{2}(s=1,2)$. Whereas, if $c_{1}^{2}+\sigma c_{2}^{2}<0$, the peak value for $\left|u_{s}[1]\right|^{2}$ is $4 \lambda_{1 I}^{2} c_{s}^{2} / r_{1}^{2}$ $(s=1,2)$.

Particularly, if $\ln \left(r_{1}\right)=\delta_{1 R}$, one can obtain a rational soliton solution of the form

$$
u_{s}[1]=\frac{2 c_{s} \lambda_{1 R} \mathrm{e}^{-\mathrm{i}\left(2 x / \lambda_{1 R}^{2}+\lambda_{1 R}^{2} t / 2\right)}}{r_{1}\left[\mathrm{i}\left(4 x / \lambda_{1 R}^{2}-\lambda_{1 R}^{2} t\right)-1\right]} .
$$

as $\lambda_{1 I} \rightarrow 0$. On the other hand, if $\sigma=-1, \ln \left(r_{1}\right)=\delta_{1 R}$, one has a rational soliton solution

$$
u_{s}[1]=\frac{2 c_{s} \lambda_{1 I} \mathrm{i}^{\mathrm{i}\left(2 x / \lambda_{1 I}^{2}+\lambda_{1 I}^{2} t / 2\right)}}{r_{1}\left[\mathrm{i}\left(4 x / \lambda_{1 I}^{2}-\lambda_{1 I}^{2} t\right)+1\right]} .
$$

as $\lambda_{1 R} \rightarrow 0$. It can be shown that the rational soliton is also of the bell shape.

\section{B. Multi-bright soliton solutions}

Letting $\left|y_{i}\right\rangle=\Phi_{1}\left(\lambda_{i}\right)\left(1, c_{i, 1}, c_{i, 2}\right)^{T}$, we can obtain

$$
m_{i, j}=\frac{2 z_{i}^{*} z_{j}}{z_{j}-z_{i}^{*}}\left[\lambda_{j} \mathrm{e}^{\theta_{i}^{*}+\theta_{j}}+\lambda_{i}^{*} \gamma_{i, j} \mathrm{e}^{-\theta_{i}^{*}-\theta_{j}}\right], \gamma_{i, j}=-\left(c_{i, 1}^{*} c_{j, 1}+\sigma c_{i, 2}^{*} c_{j, 2}\right)
$$

and

$$
\begin{aligned}
\varphi_{i} & =\mathrm{e}^{\theta_{i}}, \phi_{i}=c_{i, 1} \mathrm{e}^{-\theta_{i}}, \chi_{i}=c_{i, 2} \mathrm{e}^{-\theta_{i}}, \\
\theta_{i} & =\mathrm{i}\left(z_{i} x+\frac{1}{4 z_{i}} t\right) \equiv-z_{i, I}\left(x-v_{i} t\right)+\mathrm{i} z_{i, R}\left(x+v_{i} t\right), \quad v_{i}=\frac{1}{4\left|z_{i}\right|^{2}}, z_{i}=z_{i, R}+\mathrm{i} z_{i, I}, \quad z_{i, I}<0 .
\end{aligned}
$$

Based on the formula (30), we could derive the $N$-bright soliton solution formula:

$$
u_{s}[N]=-2 \frac{\operatorname{det}\left(\begin{array}{cc}
M & Y_{1}^{\dagger} \\
Y_{2}^{[s]} & 0
\end{array}\right)}{\operatorname{det}(M)},
$$




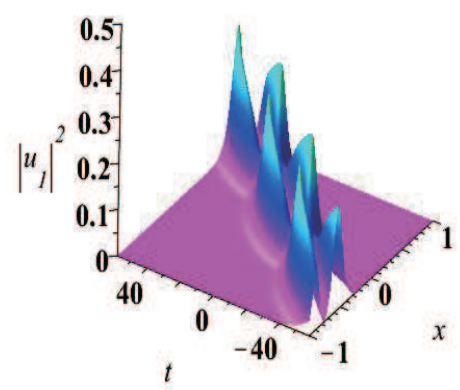

(a) $\left|u_{1}\right|^{2}$

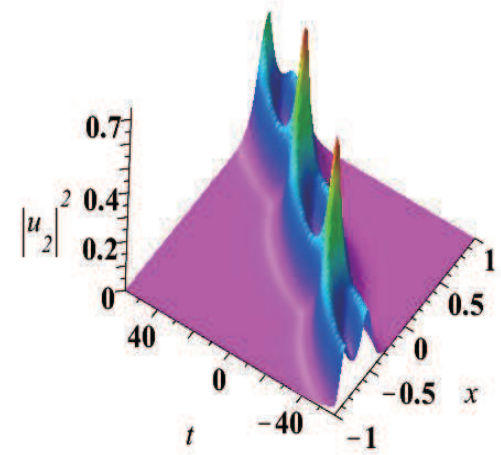

(b) $\left|u_{2}\right|^{2}$

FIG. 1: (color online): Breather solution with parameters: $\lambda_{1}=\frac{3}{10}+\frac{2}{5} \mathrm{i}, \lambda_{2}=\frac{2}{5}+\frac{3}{10} \mathrm{i}, c_{1,1}=-2, c_{2,1}=1, c_{1,2}=1, c_{2,2}=-2$.

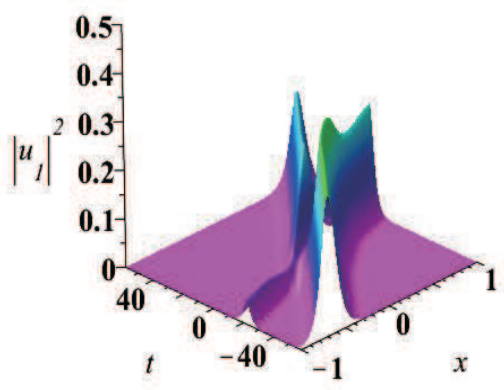

(a) $\left|u_{1}\right|^{2}$

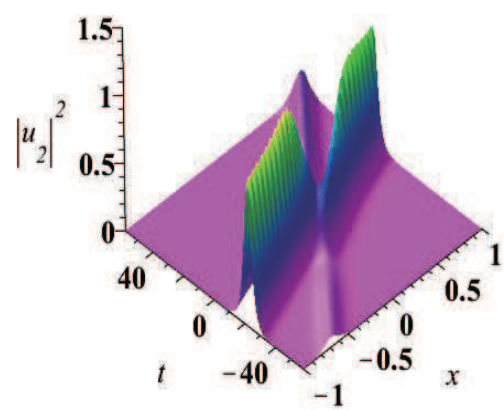

(b) $\left|u_{2}\right|^{2}$

FIG. 2: (color online): Two-soliton solution with parameters: $\lambda_{1}=\frac{3}{10}+\frac{2}{5} \mathrm{i}, \lambda_{2}=\frac{8}{15}+\frac{2}{5} \mathrm{i}, c_{1,1}=-2, c_{2,1}=1, c_{1,2}=1$, $c_{2,2}=-2$.

where $M=\left(m_{i, j}\right)_{1 \leq i, j \leq N}$ and $Y_{2}^{[s]}$ represents the $s$-th row of matrix $Y_{2}$.

To investigate the asymptotic behavior for the $N$-bright soliton solution with different velocity, we need to introduce the generalized Cauchy matrix

$$
\begin{aligned}
C\left(\Delta_{k}\right) & =\left[\frac{c_{i}^{\dagger} \Lambda c_{j}}{z_{j}-z_{i}^{*}}\right]_{i=1, j=1}^{k, k}, C\left(\Delta^{k}\right)=\left[\frac{c_{i}^{\dagger} \Lambda c_{j}}{z_{j}-z_{i}^{*}}\right]_{i=k, j=k}^{N, N}, \Lambda=\operatorname{diag}(-1,-\sigma) \\
\widehat{C}_{s}\left(\Delta_{k}\right) & =\left(\begin{array}{c}
\widehat{C}_{\text {up }} \\
\widehat{C}_{\text {lower }}
\end{array}\right), \widehat{C}_{\mathrm{up}}=\left[\frac{c_{i}^{\dagger} \Lambda c_{j}}{z_{j}-z_{i}^{*}}\right]_{i=1, j=1}^{k-1, k}, \widehat{C}_{\text {lower }}=\left(c_{1, s}, \cdots, c_{k-1, s}, c_{k, s}\right), \\
\widehat{C}_{s}\left(\Delta^{k}\right) & =\left(\begin{array}{c}
\widehat{C}^{\text {up }} \\
\widehat{C}^{\text {lower }}
\end{array}\right), \widehat{C}^{\text {lower }}=\left[\frac{c_{i}^{\dagger} \Lambda c_{j}}{z_{j}-z_{i}^{*}}\right]_{i=k+1, j=k}^{N, N}, \widehat{C}^{\text {up }}=\left(c_{k, s}, \cdots, c_{N-1, s, c_{N, s}}\right),
\end{aligned}
$$


where

$$
\begin{aligned}
& C\left(z_{k}^{c}, z_{k}^{c \dagger}\right)=\left[\frac{1}{z_{j}-z_{i}^{*}}\right]_{i=k+1, j=k+1}^{N, N}, \\
& C\left(z_{k}, z_{k}^{\dagger}\right)=\left[\frac{1}{z_{j}-z_{i}^{*}}\right]_{i=1, j=1}^{k, k}, \\
& \mathbf{c}_{k}=\left(c_{i}\right)_{i=1}^{k} \in \mathbb{C}^{k}, c_{i}=z_{i}\left(\begin{array}{c}
c_{i, 1} \\
c_{i, 2}
\end{array}\right) .
\end{aligned}
$$

Proposition 2 When $t \rightarrow \pm \infty$, the asymptotic of the $N$-soliton solution is

$$
u_{s}[N]=\sum_{k=1}^{N} u_{s, \pm}^{[k]}+O\left(\mathrm{e}^{-c|t|}\right)
$$

where $c=\min _{1,2, \cdots, N}\left(\left|z_{i, I}\right|\right) \min _{i \neq j}\left(\left|v_{i}-v_{j}\right|\right)$, the expressions of $u_{s, \pm}^{[k]}$ are given by equations (39) and (40).

Proof: Assuming $v_{1}<v_{2}<\cdots<v_{N}$, we have $\theta_{1}, \theta_{2}, \cdots, \theta_{k-1} \rightarrow-\infty ; \theta_{k+1}, \theta_{k+2}, \cdots, \theta_{N} \rightarrow+\infty$ along the trajectory $x-v_{k} t=$ const as $t \rightarrow-\infty$. On the other hand, $u_{s}[N]$ can be rewritten as

$$
u_{s}[N]=-\frac{\operatorname{det}\left(\widehat{G}_{s}\right)}{\operatorname{det}(\widehat{M})}
$$

where

$$
\begin{aligned}
& \widehat{M}=\left(\frac{z_{i}^{*} z_{j}}{z_{j}-z_{i}^{*}}\left[\lambda_{j} \mathrm{e}^{2\left(\theta_{i}^{*}+\theta_{j}\right)}+\lambda_{i}^{*} \gamma_{i, j}\right]\right)_{1 \leq i, j \leq N}, \widehat{G}_{s}=\left[\begin{array}{cc}
\widehat{M} & \widehat{Y}_{1}^{\dagger} \\
\widehat{Y}_{2}^{[s]} & 0
\end{array}\right], \\
& \widehat{Y_{1}}=\left[\begin{array}{llll}
\mathrm{e}^{2 \theta_{1}} & \mathrm{e}^{2 \theta_{2}} & \cdots & \mathrm{e}^{2 \theta_{N}}
\end{array}\right], \widehat{Y_{2}}=\left[\begin{array}{llll}
c_{1,1} & c_{2,1} & \cdots & c_{N, 1} \\
c_{1,2} & c_{2,2} & \cdots & c_{N, 2}
\end{array}\right] .
\end{aligned}
$$

It then follows

$$
\begin{aligned}
\operatorname{det}(\widehat{M}) & =\mathrm{e}^{4 \operatorname{Re}\left(\theta_{k+1}+\theta_{k+2}+\cdots+\theta_{N}\right)}\left[\operatorname{det}\left(M_{k}\right)+O\left(\mathrm{e}^{-c|t|}\right)\right], \\
\operatorname{det}\left(\widehat{G}_{s}\right) & =\mathrm{e}^{4 \operatorname{Re}\left(\theta_{k+1}+\theta_{k+2}+\cdots+\theta_{N}\right)}\left[\operatorname{det}\left(G_{k}^{[s]}\right)+O\left(\mathrm{e}^{-c|t|}\right)\right],
\end{aligned}
$$

where

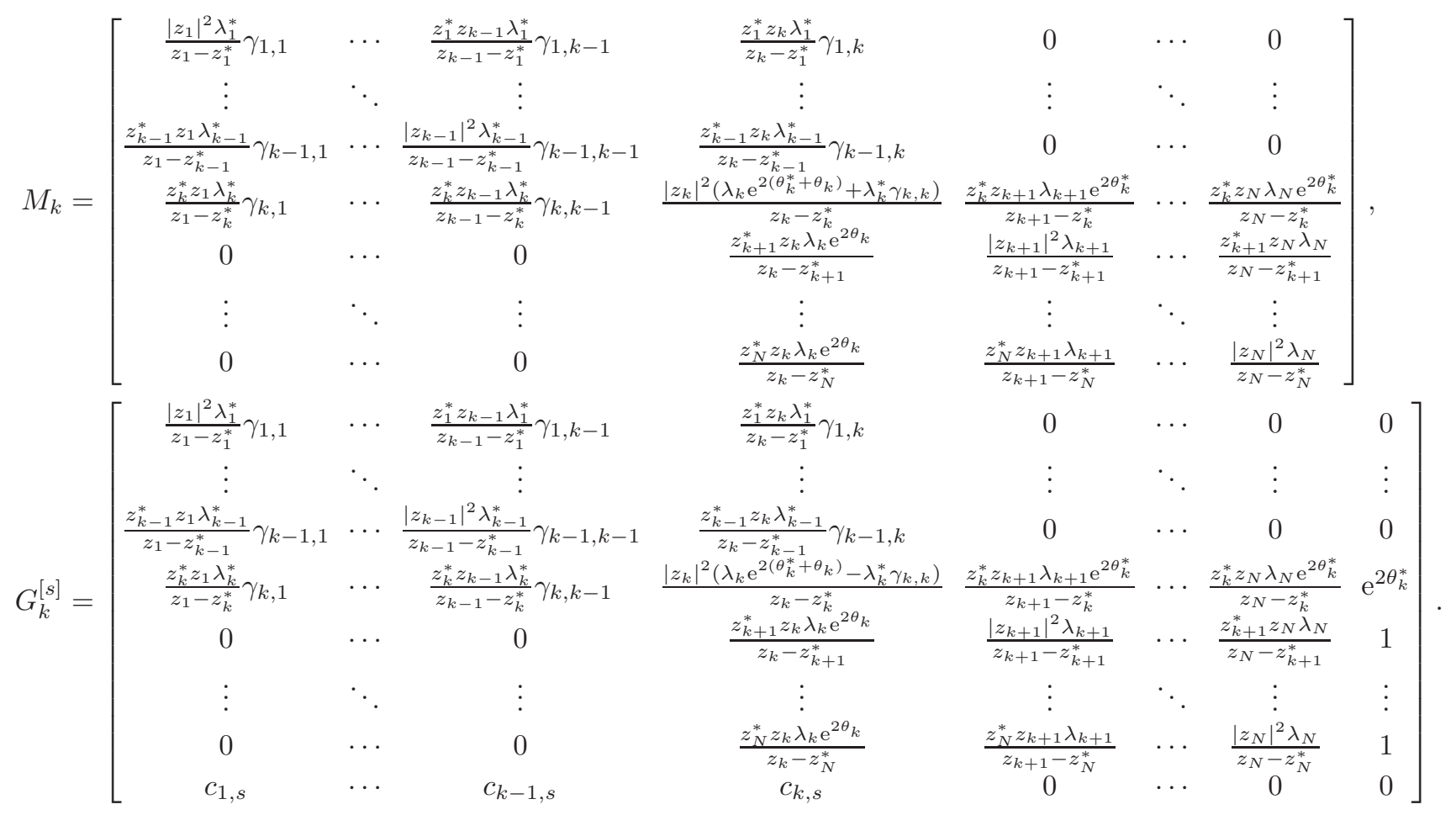


By direct calculation, we have

$$
\begin{aligned}
& \operatorname{det}\left(G_{k}^{[s]}\right)=(-1)^{k+N+1}\left|\begin{array}{cccc}
\frac{\left|z_{1}\right|^{2} \lambda_{1}^{*}}{z_{1}-z_{1}^{*}} \gamma_{1,1} & \cdots & \frac{z_{1}^{*} z_{k-1} \lambda_{1}^{*}}{z_{k-1}-z_{1}^{*}} \gamma_{1, k-1} & \frac{z_{1}^{*} z_{k} \lambda_{1}^{*}}{z_{k}-z_{1}^{*}} \gamma_{1, k} \\
\vdots & \ddots & \vdots & \vdots \\
\frac{z_{k-1}^{*} z_{1} \lambda_{k-1}^{*}}{z_{1}-z_{k-1}^{*}} \gamma_{k-1,1} & \cdots & \frac{\left|z_{k-1}\right|^{2} \lambda_{k-1}^{*}}{z_{k-1}-z_{k-1}^{*}} \gamma_{k-1, k-1} & \frac{z_{k-1}^{*} z_{k} \lambda_{k-1}^{*}}{z_{k}-z_{k-1}^{*}} \gamma_{k-1, k} \\
c_{1, s} & \cdots & c_{k-1, s} & c_{k, s}
\end{array}\right|\left|\begin{array}{cccc}
\frac{z_{k}^{*} z_{k+1} \lambda_{k+1} \mathrm{e}^{2 \theta_{k}^{*}}}{z_{k+1}-z_{k}^{*}} & \cdots & \frac{z_{k}^{*} z_{N} \lambda_{N} \mathrm{e}^{2 \theta_{k}^{*}}}{z_{N}-z_{k}^{*}} & \mathrm{e}^{2 \theta_{k}^{*}} \\
\frac{\left|z_{k+1}\right|_{k+1}}{z_{k+1}-z_{k+1}^{*}} & \cdots & \frac{z_{k+1}^{*} z_{N} \lambda_{N}}{z_{N}-z_{k+1}^{*}} & 1 \\
\vdots & \ddots & \vdots & \vdots \\
\frac{z_{N}^{*} z_{k+1} \lambda_{k+1}}{z_{k+1}-z_{N}^{*}} & \cdots & \frac{\left|z_{N}\right|^{2} \lambda_{N}}{z_{N}-z_{N}^{*}} & 1
\end{array}\right| \\
& =-\prod_{j=1}^{k-1} \lambda_{j}^{*} \operatorname{det}\left(\widehat{C}_{s}\left(\Delta_{k}\right)\right) \prod_{j=k+1}^{N}\left(\lambda_{j} z_{j}^{2} \frac{z_{j}^{*}-z_{k}^{*}}{z_{j}-z_{k}^{*}}\right) \operatorname{det}\left(C\left(\mathbf{z}_{k}^{c}, \mathbf{z}_{k}^{c \dagger}\right)\right) \mathrm{e}^{2 \theta_{k}^{*}},
\end{aligned}
$$

and

$$
\begin{aligned}
& \operatorname{det}\left(M_{k}\right) \\
& =\left[\begin{array}{ccccccc}
\frac{\left|z_{1}\right|^{2} \lambda_{1}^{*}}{z_{1}-z_{1}^{*}} \gamma_{1,1} & \cdots & \frac{z_{1}^{*} z_{k-1} \lambda_{1}^{*}}{z_{k-1}-z_{1}^{*}} \gamma_{1, k-1} & 0 & 0 & \cdots & 0 \\
\vdots & \ddots & \vdots & \vdots & \vdots & \ddots & \vdots \\
\frac{z_{k-1}^{*} z_{1} \lambda_{k-1}^{*}}{z_{1}-z_{k-1}^{*}} \gamma_{k-1,1} & \cdots & \frac{\left|z_{k-1}\right|^{2} \lambda_{k-1}^{*}}{z_{k-1}-z_{k-1}^{*}} \gamma_{k-1, k-1} & 0 & 0 & \cdots & 0 \\
\frac{z_{k}^{*} z_{1} \lambda_{k}^{*}}{z_{1}-z_{k}^{*}} \gamma_{k, 1} & \cdots & \frac{z_{k}^{*} z_{k-1} \lambda_{k}^{*}}{z_{k-1}-z_{k}^{*}} \gamma_{k, k-1} & \frac{\left|z_{k}\right|^{2} \lambda_{k} \mathrm{e}^{2\left(\theta_{k}^{*}+\theta_{k}\right)}}{z_{k}-z_{k}^{*}} & \frac{z_{k}^{*} z_{k+1} \lambda_{k+1} \mathrm{e}^{2 \theta_{k}^{*}}}{z_{k+1}-z_{k}^{*}} & \cdots & \frac{z_{k}^{*} z_{N} \lambda_{N} \mathrm{e}^{2 \theta_{k}^{*}}}{z_{N}-z_{k}^{*}} \\
0 & \cdots & 0 & \frac{z_{k+1}^{*} z_{k} \lambda_{k} \mathrm{e}^{2 \theta_{k}}}{z_{k}-z_{k+1}^{*}} & \frac{\left|z_{k+1}\right|^{2} \lambda_{k+1}}{z_{k+1}-z_{k+1}^{*}} & \cdots & \frac{z_{k+1}^{*} z_{N} \lambda_{N}}{z_{N}-z_{k+1}^{*}} \\
\vdots & \ddots & \vdots & \vdots & \vdots & \ddots & \vdots \\
0 & \cdots & 0 & \frac{z_{N}^{*} z_{k} \lambda_{k} \mathrm{e}^{2 \theta_{k}}}{z_{k}-z_{N}^{*}} & \frac{z_{N}^{*} z_{k+1} \lambda_{k+1}}{z_{k+1}-z_{N}^{*}} & \cdots & \frac{\left|z_{N}\right|^{2} \lambda_{N}}{z_{N}-z_{N}^{*}}
\end{array}\right] \\
& +\left[\begin{array}{ccccccc}
\frac{\left|z_{1}\right|^{2} \lambda_{1}^{*}}{z_{1}-z_{1}^{*}} \gamma_{1,1} & \cdots & \frac{z_{1}^{*} z_{k-1} \lambda_{1}^{*}}{z_{k-1}-z_{1}^{*}} \gamma_{1, k-1} & \frac{z_{1}^{*} z_{k} \lambda_{1}^{*}}{z_{k}-z_{1}^{*}} \gamma_{1, k} & 0 & \cdots & 0 \\
\vdots & \ddots & \vdots & \vdots & \vdots & \ddots & \vdots \\
\frac{z_{k-1}^{*} z_{1} \lambda_{k-1}^{*}}{z_{1}-z_{k-1}^{*}} \gamma_{k-1,1} & \cdots & \frac{\left|z_{k-1}\right|^{2} \lambda_{k-1}^{*}}{z_{k-1}-z_{k-1}^{*}} \gamma_{k-1, k-1} & \frac{z_{k-1}^{*} z_{k} \lambda_{k-1}^{*}}{z_{k}-z_{k-1}^{*}} \gamma_{k-1, k} & 0 & \cdots & 0 \\
\frac{z_{k}^{*} z_{1} \lambda_{k}^{*}}{z_{1}-z_{k}^{*}} \gamma_{k, 1} & \cdots & \frac{z_{k}^{*} z_{k-1} \lambda_{k}^{*}}{z_{k-1}-z_{k}^{*}} \gamma_{k, k-1} & \frac{\left|z_{k}\right|^{2} \lambda_{k}^{*} \gamma_{k, k}}{z_{k}-z_{k}^{*}} & \frac{z_{k}^{*} z_{k+1} \lambda_{k+1} \mathrm{e}^{2 \theta_{k}^{*}}}{z_{k+1}-z_{k}^{*}} & \cdots & \frac{z_{k}^{*} z_{N} \lambda_{N} \mathrm{e}^{2 \theta_{k}^{*}}}{z_{N}-z_{k}^{*}} \\
0 & \cdots & 0 & 0 & \frac{\mid z_{k+1} \lambda_{k+1}}{z_{k+1}-z_{k+1}^{*}} & \cdots & \frac{z_{k+1}^{*} z_{N} \lambda_{N}}{z_{N}-z_{k+1}^{*}} \\
\vdots & \ddots & \vdots & \vdots & \vdots & \ddots & \vdots \\
0 & \cdots & 0 & 0 & \frac{z_{N}^{*} z_{k+1} \lambda_{k+1}}{z_{k+1}-z_{N}^{*}} & \cdots & \frac{\left|z_{N}\right|^{2} \lambda_{N}}{z_{N}-z_{N}^{*}}
\end{array}\right] \\
& =\operatorname{det}\left(C\left(\mathbf{z}_{k}^{c}, \mathbf{z}_{k}^{c \dagger}\right)\right) \prod_{j=1}^{k-1} \lambda_{j}^{*} \prod_{j=k+1}^{N} \lambda_{j}\left|z_{j}\right|^{2}\left[\frac{\left|z_{k}\right|^{2} \lambda_{k} \operatorname{det}\left(C\left(\Delta_{k-1}\right)\right)}{z_{k}-z_{k}^{*}} \prod_{j=k+1}^{N}\left|\frac{z_{k}-z_{j}}{z_{k}-z_{j}^{*}}\right|^{2} \mathrm{e}^{2\left(\theta_{k}^{*}+\theta_{k}\right)}+\lambda_{k}^{*} \operatorname{det}\left(C\left(\Delta_{k}\right)\right)\right] \text {. }
\end{aligned}
$$

Thus, along the trajectory $x-v_{k} t=$ const, we have

$$
\begin{aligned}
u_{s}[N] & =\frac{\operatorname{det}\left(\widehat{C}_{s}\left(\Delta_{k}\right)\right) \prod_{j=k+1}^{N}\left(\frac{z_{j}^{2}}{\left|z_{j}\right|^{2}} \frac{z_{j}^{*}-z_{k}^{*}}{z_{j}-z_{k}^{*}}\right) \mathrm{e}^{2 \theta_{k}^{*}}}{\frac{\left|z_{k}\right|^{2} \lambda_{k}}{z_{k}-z_{k}^{*}} \operatorname{det}\left(C\left(\Delta_{k-1}\right)\right) \prod_{j=k+1}^{N}\left|\frac{z_{k}-z_{j}}{z_{k}-z_{j}^{*}}\right|^{2} \mathrm{e}^{2\left(\theta_{k}^{*}+\theta_{k}\right)}+\lambda_{k}^{*} \operatorname{det}\left(C\left(\Delta_{k}\right)\right)}+O\left(\mathrm{e}^{-c|t|}\right) \\
& =u_{s,-}^{[k]}+O\left(\mathrm{e}^{-c|t|}\right)
\end{aligned}
$$


as $t \rightarrow-\infty$, where

$$
\begin{aligned}
u_{s,-}^{[k]} & =\hat{c}_{k, s}^{-} \frac{z_{k}-z_{k}^{*}}{\left|z_{k}\right|^{2}} \frac{\mathrm{e}^{2 \widehat{\theta}_{k,-}^{*}}}{\lambda_{k} \mathrm{e}^{2\left(\widehat{\theta}_{k,-}^{*}+\widehat{\theta}_{k,-}\right)}+\lambda_{k}^{*} \delta_{k}^{-}} \\
\widehat{\theta}_{k,-} & =\theta_{k}+\frac{1}{2} \sum_{j=k+1}^{N} \ln \left(\frac{z_{j}-z_{k}}{z_{j}^{*}-z_{k}}\right), \\
\hat{c}_{k, s}^{-} & =\frac{\operatorname{det}\left(\widehat{C}_{s}\left(\Delta_{k}\right)\right)}{\operatorname{det}\left(C\left(\Delta_{k-1}\right)\right)} \prod_{j=k+1}^{N} \frac{z_{j}^{2}}{\left|z_{j}\right|^{2}}, \\
\delta_{k}^{-} & =\frac{z_{k}-z_{k}^{*}}{\left|z_{k}\right|^{2}} \frac{\operatorname{det}\left(C\left(\Delta_{k}\right)\right)}{\operatorname{det}\left(C\left(\Delta_{k-1}\right)\right)} .
\end{aligned}
$$

For the general case $x-v t=$ const, $v \neq v_{k}(k=1,2, \cdots, N)$, we have $u_{s}[N]=O\left(\mathrm{e}^{-c|t|}\right)$. Thus the asymptotic behavior is analyzed as $t \rightarrow-\infty$.

By the same procedure as above, we can obtain the asymptotical behavior as $t \rightarrow+\infty$. To be specific, along the trajectory $x-v_{k} t=$ const, one has

$$
\begin{aligned}
u_{s}[N] & =\frac{\operatorname{det}\left(\widehat{C}_{s}\left(\Delta^{k}\right)\right) \prod_{j=1}^{k-1}\left(\frac{z_{j}^{2}}{\left|z_{j}\right|^{2}} \frac{z_{j}^{*}-z_{k}^{*}}{z_{j}-z_{k}^{*}}\right) \mathrm{e}^{2 \theta_{k}^{*}}}{\frac{\left|z_{k}\right|^{2} \lambda_{k}}{z_{k}-z_{k}^{*}} \operatorname{det}\left(C\left(\Delta^{k+1}\right)\right) \prod_{j=1}^{k-1}\left|\frac{z_{k}-z_{j}}{z_{k}-z_{j}^{*}}\right|^{2} \mathrm{e}^{2\left(\theta_{k}^{*}+\theta_{k}\right)}+\lambda_{k}^{*} \operatorname{det}\left(C\left(\Delta^{k}\right)\right)}+O\left(\mathrm{e}^{-c|t|}\right) \\
& =u_{s,+}^{[k]}+O\left(\mathrm{e}^{-c|t|}\right)
\end{aligned}
$$

as $t \rightarrow+\infty$, where

$$
\begin{aligned}
u_{s,+}^{[k]} & =\hat{c}_{k, s}^{+} \frac{z_{k}-z_{k}^{*}}{\left|z_{k}\right|^{2}} \frac{\mathrm{e}^{2 \widehat{\theta}_{k,+}^{*}}}{\lambda_{k} \mathrm{e}^{2\left(\widehat{\theta}_{k,+}^{*}+\widehat{\theta}_{k,+}\right)}+\lambda_{k}^{*} \delta_{k}^{+}} \\
\widehat{\theta}_{k,+} & =\theta_{k}+\frac{1}{2} \sum_{j=1}^{k-1} \ln \left(\frac{z_{j}-z_{k}}{z_{j}^{*}-z_{k}}\right) \\
\hat{c}_{k, s}^{+} & =\frac{\operatorname{det}\left(\widehat{C}_{s}\left(\Delta^{k}\right)\right)}{\operatorname{det}\left(C\left(\Delta^{k+1}\right)\right)} \prod_{j=1}^{k-1} \frac{z_{j}^{2}}{\left|z_{j}\right|^{2}}, \\
\delta_{k}^{+} & =\frac{z_{k}-z_{k}^{*}}{\left|z_{k}\right|^{2}} \frac{\operatorname{det}\left(C\left(\Delta^{k}\right)\right)}{\operatorname{det}\left(C\left(\Delta^{k+1}\right)\right)} .
\end{aligned}
$$

Consequently, the asymptotic behaviors of $N$-bright soliton's are analyzed.

\section{THE SOLITON SOLUTIONS WITH NONVANISHING BOUNDARY CONDITION}

In this section, we construct all kinds of soliton solutions from the plane wave seed solution. These soliton solutions of coupled FL equation are similar to the ones of coupled nonlinear Schrödinger (CNLS) equation, but they have much richer structures than the ones of the CNLS equation. For instance, the bright-anti-dark soliton and dark-anti-dark soliton solutions, which do not occur in the CNLS equation, exist in the coupled FL equation. We remark here that the soliton is called dark if its amplitude is lower than the background, and the soliton is called anti-dark if its amplitude is higher than the background.

To this end, we start with the plane wave solution for equation (1):

$$
u_{i}[0]=a_{i} \mathrm{e}^{\mathrm{i} \omega_{i}}, i=1,2
$$


where

$$
\begin{aligned}
& \omega_{1}=b_{1} x-\frac{1}{2}\left(2 a_{1}^{2}+\sigma a_{2}^{2}-\frac{2}{b_{1}}+\sigma a_{2}^{2} \frac{b_{2}}{b_{1}}\right) t, \\
& \omega_{2}=b_{2} x-\frac{1}{2}\left(2 \sigma a_{2}^{2}+a_{1}^{2}-\frac{2}{b_{2}}+a_{1}^{2} \frac{b_{1}}{b_{2}}\right) t,
\end{aligned}
$$

$a_{i} \in \mathbb{R}$ and $b_{1}, b_{2}$ are nonzero real parameters. Assuming $a_{1} \neq 0$ and introducing $z=1 / \lambda^{2}$, we have the fundamental solution to the system (16)

$$
\Phi_{2}(\lambda)=D_{1} E_{1} \operatorname{diag}\left(\mathrm{e}^{\vartheta_{1}}, \mathrm{e}^{\vartheta_{2}}, \mathrm{e}^{\vartheta_{3}}\right), D_{1}=\operatorname{diag}\left(1, \mathrm{e}^{\mathrm{i} \omega_{1}}, \mathrm{e}^{\mathrm{i} \omega_{2}}\right),
$$

where

$$
\vartheta_{i}=\mathrm{i}\left(\kappa_{i}-z\right)\left(x+\frac{1}{2 b_{1} b_{2} z}\left(\kappa_{i}-z+b_{1}+b_{2}\right) t\right), i=1,2,3 .
$$

Then we have the following conclusions

- If $b_{1}=b_{2}$, then

$$
E_{1}=\left(\begin{array}{ccc}
1 & 1 & 0 \\
\frac{a_{1} b_{1}}{\lambda\left(\kappa_{1}+b_{1}\right)} & \frac{a_{1} b_{1}}{\lambda\left(\kappa_{2}+b_{1}\right)} & -\frac{a_{2}}{a_{1}} \\
\frac{a_{2} b_{1}}{\lambda\left(\kappa_{1}+b_{1}\right)} & \frac{a_{2} b_{1}}{\lambda\left(\kappa_{2}+b_{1}\right)} & 1
\end{array}\right)
$$

where $\kappa_{i},(i=1,2)$ satisfies the following equation

$$
(\kappa / z-2)\left(\kappa+b_{1}\right)+\left(a_{1}^{2}+\sigma a_{2}^{2}\right) b_{1}^{2}=0,
$$

and $\kappa_{3}=-b_{1}$.

- If $b_{1} \neq b_{2}$ and $a_{2} \neq 0$, then

$$
E_{1}=\left(\begin{array}{ccc}
1 & 1 & 1 \\
\frac{a_{1} b_{1}}{\lambda\left(\kappa_{1}+b_{1}\right)} & \frac{a_{1} b_{1}}{\lambda\left(\kappa_{2}+b_{1}\right)} & \frac{a_{1} b_{1}}{\lambda\left(\kappa_{3}+b_{1}\right)} \\
\frac{a_{2} b_{2}}{\lambda\left(\kappa_{1}+b_{2}\right)} & \frac{a_{2} b_{2}}{\lambda\left(\kappa_{2}+b_{2}\right)} & \frac{a_{2} b_{2}}{\lambda\left(\kappa_{3}+b_{2}\right)}
\end{array}\right),
$$

where $\kappa_{i},(i=1,2,3)$ satisfies the following equation

$$
(\kappa / z-2)\left(\kappa+b_{1}\right)\left(\kappa+b_{2}\right)+a_{1}^{2} b_{1}^{2}\left(\kappa+b_{2}\right)+\sigma a_{2}^{2} b_{2}^{2}\left(\kappa+b_{1}\right)=0 .
$$

Based on the roots of characteristic equation (43) and (44), we can classify the parameters into four cases. Here we denote $z=z_{1}=\lambda_{1}^{-2}$.

Case (a): $\Omega_{1} \equiv\left\{a_{1}, a_{2}, b_{1}, b_{2} \mid b_{1}=b_{2}, a_{1} \neq 0,2 b_{1}-\left(a_{1}^{2}+\sigma a_{2}^{2}\right) b_{1}^{2}=0\right\}$. In this case, the root for the characteristic equation (43) can be solved as

$$
\kappa_{1}=2 z_{1}-b_{1}, \kappa_{2}=0, \kappa_{3}=-b_{1} .
$$

Case (b): $\Omega_{2} \equiv\left\{a_{1}, a_{2}, b_{1}, b_{2} \mid b_{1}=b_{2}, a_{1} \neq 0,2 b_{1}-\left(a_{1}^{2}+\sigma a_{2}^{2}\right) b_{1}^{2} \neq 0\right\}$. In this case, to avoid the radical expression for the root, we introduce a variable $\xi_{1} \in \mathbb{C}$. Denote $\gamma_{1}=b_{1}-\left(a_{1}^{2}+\sigma a_{2}^{2}\right) b_{1}^{2}, \delta_{1}=\left|b_{1}\right| \sqrt{\left|a_{1}^{2}+\sigma a_{2}^{2} \| \gamma_{1}+b_{1}\right|}$, we then have

$$
\begin{aligned}
& z_{1}= \begin{cases}\frac{\delta_{1}}{4}\left(\xi_{1}-\xi_{1}^{-1}\right)-\frac{\gamma_{1}}{2}, & \text { if }\left(\gamma_{1}+b_{1}\right)\left(a_{1}^{2}+\sigma a_{2}^{2}\right)>0, \\
\frac{\delta_{1}}{4}\left(\xi_{1}+\xi_{1}^{-1}\right)-\frac{\gamma_{1}}{2}, & \text { if }\left(\gamma_{1}+b_{1}\right)\left(a_{1}^{2}+\sigma a_{2}^{2}\right)<0,\end{cases} \\
& \kappa_{1}=-\frac{b_{1}+\gamma_{1}}{2}+\frac{\delta_{1}}{2} \xi_{1}, \\
& \kappa_{2}=-\frac{b_{1}+\gamma_{1}}{2} \mp \frac{\delta_{1}}{2} \xi_{1}^{-1}, \quad \kappa_{3}=-b_{1} .
\end{aligned}
$$


Case (c): $\Omega_{3} \equiv\left\{a_{1}, a_{2}, b_{1}, b_{2} \mid a_{1}, a_{2} \neq 0, \sigma b_{2}=2 a_{2}^{-2}-b_{1} a_{1}^{2} a_{2}^{-2}\right\}$. In this case, we introduce a variable $\xi_{2} \in \mathbb{C}$ for the same reason as case (b). Denote $\delta_{2}=\frac{a_{1}^{2}\left|\left(a_{1}^{2}+\sigma a_{2}^{2}\right) b_{1}-2\right|}{a_{2}^{2}} \sqrt{\left|b_{1}\left(2 / a_{1}^{2}-b_{1}\right)\right|}, \gamma_{2}=-\frac{\left(1-a_{1}^{2} b_{1}\right)\left(\left(a_{1}^{2}+\sigma a_{2}^{2}\right) b_{1}-2\right)}{2 a_{2}^{2}}$, it then follows

$$
\begin{aligned}
& z_{1}= \begin{cases}\frac{\delta_{2}}{4}\left(\xi_{2}-\xi_{2}^{-1}\right)+\gamma_{2}, & \text { if } b_{1}\left(2 / a_{1}^{2}-b_{1}\right)>0, \\
\frac{\delta_{2}}{4}\left(\xi_{2}+\xi_{2}^{-1}\right)+\gamma_{2}, & \text { if } b_{1}\left(2 / a_{1}^{2}-b_{1}\right)<0,\end{cases} \\
& \kappa_{1}=-\frac{b_{1}+b_{2}}{2}+\gamma_{2}+\frac{\delta_{2}}{2} \xi_{2}, \\
& \kappa_{2}=-\frac{b_{1}+b_{2}}{2}+\gamma_{2} \mp \frac{\delta_{2}}{2} \xi_{2}^{-1}, \quad \kappa_{3}=0 .
\end{aligned}
$$

Case (d): $\Omega_{4} \equiv\left\{a_{1}, a_{2}, b_{1}, b_{2} \mid a_{1}, a_{2} \neq 0, \sigma b_{2} \neq 2 a_{2}^{-2}-b_{1} a_{1}^{2} a_{2}^{-2}, b_{1} \neq b_{2}\right\}$. In this case, the radical expression for the roots is we unavoidable. A direct way of obtaining the roots is to use the Kardan formula. However, one often turns to find numerical solutions for the roots to avoid the complicated formula.

Remark 1 The relations between the roots and coefficients for the characteristic equation (44) are

$$
\begin{aligned}
\sum_{i=1}^{3} \kappa_{i}+b_{1}+b_{2} & =2 z_{1}, \\
\prod_{i=1}^{3}\left(\kappa_{i}+b_{1}\right) & =a_{1}^{2} b_{1}^{2}\left(b_{1}-b_{2}\right)\left(\sum_{i=1}^{3} \kappa_{i}+b_{1}+b_{2}\right), \\
\prod_{i=1}^{3}\left(\kappa_{i}+b_{2}\right) & =-\sigma a_{2}^{2} b_{2}^{2}\left(b_{1}-b_{2}\right)\left(\sum_{i=1}^{3} \kappa_{i}+b_{1}+b_{2}\right) .
\end{aligned}
$$

In what follows, we will use the formulas (27), (41) and (42) to construct single soliton solutions. Generally, the single soliton solutions can be represented as

$$
u_{1}[1]=a_{1}\left[\frac{\beta+\frac{2 \psi_{1} \varphi_{1}^{*}}{a_{1}} \mathrm{e}^{-\mathrm{i} \omega_{1}}}{\beta}\right] \mathrm{e}^{\mathrm{i} \omega_{1}}, u_{2}[1]=a_{2}\left[\frac{\frac{\beta+2 \chi_{1} \varphi_{1}^{*}}{a_{2}} \mathrm{e}^{-\mathrm{i} \omega_{2}}}{\beta}\right] \mathrm{e}^{\mathrm{i} \omega_{2}} .
$$

Prior to calculating the explicit form for $\beta$, we need the following proposition.

Proposition 3 If we choose a special solution $\left|y_{1}\right\rangle=\left(\varphi_{1}, \psi_{1}, \chi_{1}\right)^{\mathrm{T}} \equiv \Phi_{2}\left(\lambda_{1}\right)\left(c_{1}, c_{2}, c_{3}\right)^{\mathrm{T}}$, then

$$
\beta=2 \lambda_{1}^{-1} M_{1}, M_{1}=Z_{l, m}: c_{l}^{*} c_{m} \mathrm{e}^{\vartheta_{l}^{*}+\vartheta_{m}} \equiv \sum_{l=1}^{3} \sum_{m=1}^{3} Z_{l, m} c_{l}^{*} c_{m} \mathrm{e}^{\vartheta_{l}^{*}+\vartheta_{m}},
$$

where $Z_{l, m}$ are of the following forms depending on four cases mentioned previously.

- If $a_{1}, a_{2}, b_{1}, b_{2} \in \Omega_{1}$, then

$$
Z_{l, m}= \begin{cases}\frac{\kappa_{l}^{*}}{\kappa_{m}-\kappa_{l}^{*}} & \text { if } 1 \leq l \leq 2,1 \leq m \leq 2, l+m<4, \\ \frac{\left|z_{1}\right|^{2}\left(\left(a_{1}^{2}+\sigma a_{2}^{2}\right) z_{1}-1\right)}{\left|z_{1}\right|^{2}-z_{1}^{2}} & \text { if } l=m=2, \\ \frac{\left(\sigma+\frac{a_{2}^{2}}{a_{1}^{2}}\right)\left|z_{1}\right|}{z_{1}^{*}-z_{1}} & \text { if } l=m=3, \\ 0 & \text { otherwise. }\end{cases}
$$

- If $a_{1}, a_{2}, b_{1}, b_{2} \in \Omega_{2}$, then

$$
Z_{l, m}= \begin{cases}\frac{\kappa_{l}^{*}}{\kappa_{m}-\kappa_{l}^{*}} & \text { if } 1 \leq l \leq 2,1 \leq m \leq 2, \\ \frac{\left(\sigma+\frac{a_{2}^{2}}{a_{1}^{2}}\right)\left|z_{1}\right|}{z_{1}^{*}-z_{1}} & \text { if } l=m=3, \\ 0 & \text { otherwise. }\end{cases}
$$


- If $a_{1}, a_{2}, b_{1}, b_{2} \in \Omega_{3}$, then

$$
Z_{l, m}= \begin{cases}\frac{\left|z_{1}\right|^{2}\left(\left(a_{1}^{2}+\sigma a_{2}^{2}\right) z_{1}-1\right)}{\left|z_{1}\right|^{2}-z_{1}^{2}} & \text { if } l=m=3, \\ \frac{\kappa_{l}^{*}}{\kappa_{m}-\kappa_{l}^{*}} & \text { otherwise. }\end{cases}
$$

- If $a_{1}, a_{2}, b_{1}, b_{2} \in \Omega_{4}$, then

$$
Z_{l, m}=\frac{\kappa_{l}^{*}}{\kappa_{m}-\kappa_{l}^{*}}
$$

Proof: Firstly, we have

$$
\frac{\kappa_{m}}{z_{1}}-2+\frac{a_{1}^{2} b_{1}^{2}}{\kappa_{m}+b_{1}}+\frac{\sigma a_{2}^{2} b_{2}^{2}}{\kappa_{m}+b_{2}}=0
$$

and its complex conjugate

$$
\frac{\kappa_{l}^{*}}{z_{1}^{*}}-2+\frac{a_{1}^{2} b_{1}^{2}}{\kappa_{l}^{*}+b_{1}}+\frac{\sigma a_{2}^{2} b_{2}^{2}}{\kappa_{l}^{*}+b_{2}}=0 .
$$

Subtracting equation (55) from (54) yields

$$
\frac{\lambda_{1}^{2} \kappa_{m}-\lambda_{1}^{* 2} \kappa_{l}^{*}}{\left(\kappa_{l}^{*}-\kappa_{m}\right)}+\frac{a_{1}^{2} b_{1}^{2}}{\left(\kappa_{m}+b_{1}\right)\left(\kappa_{l}^{*}+b_{1}\right)}+\frac{\sigma a_{2}^{2} b_{2}^{2}}{\left(\kappa_{m}+b_{2}\right)\left(\kappa_{l}^{*}+b_{2}\right)}=0 .
$$

The coefficient of $\mathrm{e}^{\vartheta_{m}+\vartheta_{l}^{*}}$ can be simplified as

$$
\begin{aligned}
& \frac{2 c_{l} c_{m}^{*}}{\lambda_{1}^{* 2}-\lambda_{1}^{2}}\left[\lambda_{1}-\frac{\lambda_{1}^{*}}{\lambda_{1} \lambda_{1}^{*}}\left(\frac{a_{1}^{2} b_{1}^{2}}{\left(\kappa_{m}+b_{1}\right)\left(\kappa_{l}^{*}+b_{1}\right)}+\frac{\sigma a_{2}^{2} b_{2}^{2}}{\left(\kappa_{m}+b_{2}\right)\left(\kappa_{l}^{*}+b_{2}\right)}\right)\right] \\
= & \frac{2 c_{l} c_{m}^{*}}{\lambda_{1}^{* 2}-\lambda_{1}^{2}}\left[\frac{\lambda_{1}^{*}\left(\lambda_{1}^{2} \kappa_{m}-\lambda_{1}^{* 2} \kappa_{l}^{*}\right)+\lambda_{1}^{2} \lambda_{1}^{*}\left(\kappa_{l}^{*}-\kappa_{m}\right)}{\lambda_{1} \lambda_{1}^{*}\left(\kappa_{l}^{*}-\kappa_{m}\right)}\right] \\
= & \frac{2 c_{l} c_{m}^{*} \kappa_{l}^{*}}{\lambda_{1}\left(\kappa_{m}-\kappa_{l}^{*}\right)}
\end{aligned}
$$

by referring equation (56). The four cases can be proved by analyzing above relation by tedious work, which is omitted here.

We comment here that if $\kappa_{i} \in \mathbb{R}$, we can not use the above relation. Instead, we have to use the definition to derive the relation directly.

\section{A. Bright-dark/Bright-anti-dark solution}

First, we consider $a_{1}, a_{2}, b_{1}, b_{2} \in \Omega_{1}$ and $a_{2}=0$. Under this case, one can obtain the following general solution by formula (48)

$$
u_{1}[1]=a_{1}\left(\frac{N_{1}^{[1]}}{M_{1}}\right) \mathrm{e}^{\mathrm{i} \omega_{1}}, u_{2}[1]=\frac{N_{2}^{[1]}}{M_{1}} \mathrm{e}^{\mathrm{i} \omega_{1}}
$$

where

$$
\begin{aligned}
& N_{1}^{[1]}=\sum_{l, m=1, l+m<4}^{2,2} \frac{\kappa_{l}^{*}+b_{1}}{\kappa_{m}+b_{1}} \frac{c_{m} c_{l}^{*} \kappa_{m} \mathrm{e}^{\vartheta_{m}+\vartheta_{l}^{*}}}{\kappa_{m}-\kappa_{l}^{*}}+\frac{\left|c_{2}\right|^{2} z_{1}^{2}\left(a_{1}^{2} z_{1}^{*}-1\right)}{\left|z_{1}\right|^{2}-z_{1}^{2}} \mathrm{e}^{\vartheta_{2}+\vartheta_{2}^{*}}+\frac{\sigma\left|c_{3}\right|^{2}\left|z_{1}\right|}{\left(z_{1}^{*}-z_{1}\right)} \mathrm{e}^{\vartheta_{3}+\vartheta_{3}^{*}}, \\
& N_{2}^{[1]}=c_{3} \lambda_{1}\left(c_{1}^{*} \mathrm{e}^{\vartheta_{3}+\vartheta_{1}^{*}}+c_{2}^{*} \mathrm{e}^{\vartheta_{3}+\vartheta_{2}^{*}}\right) .
\end{aligned}
$$

If $c_{1}=0, c_{2} c_{3} \neq 0$, the solution $\left|u_{1}[1]\right|^{2}=a_{1}^{2}$ and $\left|u_{2}[1]\right|^{2}$ is a bright soliton along the line $t=$ const. If $c_{2}=0$, $c_{1} c_{3} \neq 0$, the solution is a bright-dark soliton. If $c_{3}=0, c_{1} c_{2} \neq 0$, then the solution $\left|u_{1}[1]\right|^{2}$ is a breather solution and 


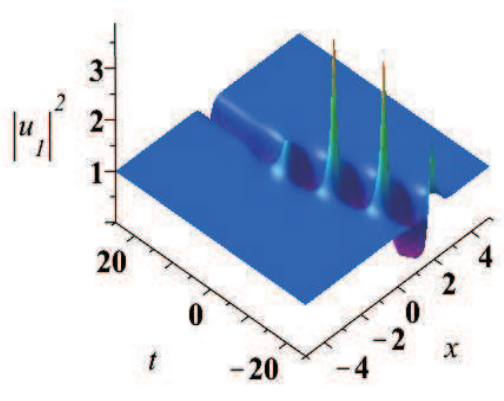

(a) $\left|u_{1}\right|^{2}$

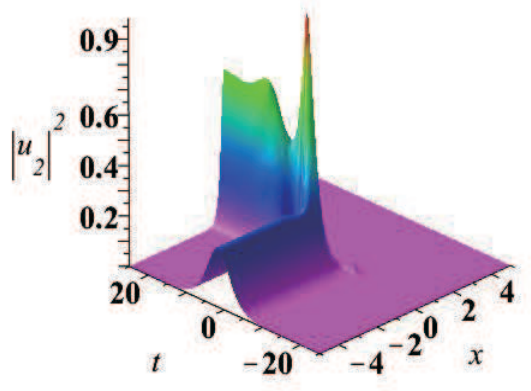

(b) $\left|u_{2}\right|^{2}$

FIG. 3: (color online): Resonant bright-dark-breather solution with parameters: $a_{1}=1, b_{1}=b_{2}=2, \lambda_{1}=\frac{1}{2}(1+\mathrm{i}), z_{1}=-2 \mathrm{i}$, $\kappa_{1}=-2-4 \mathrm{i}, \kappa_{2}=0, c_{1}=c_{2}=c_{3}=1$.

$u_{2}[1]=0$. If $c_{1} c_{2} c_{3} \neq 0$, the solution is a resonant bright-dark-breather solution which can be viewed as the nonlinear superposition of above three types of solutions. Then we consider the case $a_{1}, a_{2}, b_{1}, b_{2} \in \Omega_{2}$ and $a_{2}=0$, under which we can obtain the following general solution by formula (48)

$$
\begin{aligned}
& u_{1}[1]=a_{1}\left(\frac{N_{1}^{[2]}}{M_{1}}\right) \mathrm{e}^{\mathrm{i} \omega_{1},}, \\
& u_{2}[1]=\left(\frac{N_{2}^{[2]}}{M_{1}}\right) \mathrm{e}^{\mathrm{i} \omega_{1}},
\end{aligned}
$$

where

$$
\begin{aligned}
& N_{1}^{[2]}=\sum_{l, m=1}^{2} \frac{\kappa_{l}^{*}+b_{1}}{\kappa_{m}+b_{1}} \frac{c_{m} c_{l}^{*} \kappa_{m} \mathrm{e}^{\vartheta_{m}+\vartheta_{l}^{*}}}{\kappa_{m}-\kappa_{l}^{*}}+\frac{\sigma\left|c_{3}\right|^{2}\left|z_{1}\right|}{\left(z_{1}^{*}-z_{1}\right)} \mathrm{e}^{\vartheta_{3}+\vartheta_{3}^{*}} \\
& N_{2}^{[2]}=c_{3} \lambda_{1}\left(c_{1}^{*} \mathrm{e}^{\vartheta_{3}+\vartheta_{1}^{*}}+c_{2}^{*} \mathrm{e}^{\vartheta_{3}+\vartheta_{2}^{*}}\right)
\end{aligned}
$$

and

$$
\vartheta_{i}=\mathrm{i}\left(\kappa_{i}-z_{1}\right)\left[x+\frac{z_{1}^{*}}{2 b_{1} b_{2}\left|z_{1}\right|^{2}}\left(\kappa_{i}-z_{1}+2 b_{1}\right) t\right] .
$$

It can be shown that if $c_{1}=0, c_{2} c_{3} \neq 0\left(c_{2}=0, c_{1} c_{3} \neq 0\right)$, the solution is either a bright-dark soliton or a bright-antidark soliton. Particularly, when $\operatorname{sign}\left(\operatorname{Re}\left(\kappa_{1}\right) / \operatorname{Re}\left(z_{1}\right)\right)>0$, the solution is a bright-dark soliton. The peak for $\left|u_{1}[1]\right|^{2}$ is along the line $\operatorname{Re}\left(\vartheta_{3}-\vartheta_{1}\right)+\ln \left(\frac{\left|f_{1}\right|\left|z_{1}\right| \operatorname{Im}\left(\kappa_{1}\right)}{\left|d_{1}\right|\left|\kappa_{1}\right| \operatorname{Im}\left(z_{1}\right)}\right)=0$, and the peak values are $a_{1}^{2}\left[1-\frac{2 \operatorname{Im}\left(\kappa_{1}\right) \operatorname{Im}\left(\frac{\kappa_{1}}{\kappa_{1}+b_{1}}\right)}{\left|\kappa_{1}\right|-\operatorname{Re}\left(\kappa_{1}\right)}\right]$ for $\left|u_{1}[1]\right|^{2}$ and $\frac{2\left|\operatorname{Im}\left(z_{1}\right) \operatorname{Im}\left(\kappa_{1}\right)\right|}{\left|z_{1}\right|^{2}\left(\left|\kappa_{1}\right|-\operatorname{Re}\left(\kappa_{1}\right)\right)}$ for $\left|u_{2}[1]\right|^{2}$. When $\operatorname{sign}\left(\operatorname{Re}\left(\kappa_{1}\right) / \operatorname{Re}\left(z_{1}\right)\right)<0$, the solution is a bright-anti-dark soliton. The peak values are $a_{1}^{2}\left[1+\frac{2\left|\operatorname{Im}\left(\kappa_{1}\right) \operatorname{Im}\left(\frac{\kappa_{1}}{\left.\kappa_{1}+b_{1}\right)}\right)\right|}{\left|\kappa_{1}\right|+\operatorname{Re}\left(\kappa_{1}\right)}\right]\left|u_{1}[1]\right|^{2}$ and $\frac{2\left|\operatorname{Im}\left(z_{1}\right) \operatorname{Im}\left(\kappa_{1}\right)\right|}{\left|z_{1}\right|^{2}\left(\left|\kappa_{1}\right|+\operatorname{Re}\left(\kappa_{1}\right)\right)}$ for $\left|u_{2}[1]\right|^{2}$.

On the other hand, as $c_{1} c_{2} \neq 0, c_{3}=0$, one obtains a breather solution, as $c_{1} c_{2} c_{3} \neq 0$, one arrives at a resonant bright-dark-breather solution.

\section{B. Dark/anti-dark soliton}

In CNLS equation, there only exists dark soliton solution. Whereas there exists dark and anti-dark soliton solutions in the coupled FL equation (11). If we apply the formula (48) for a complex spectral parameter $\lambda_{1}$, the singularity occurs. With the aid of technique in [37], we can derive the dark/anti-dark soliton to equation (10). 


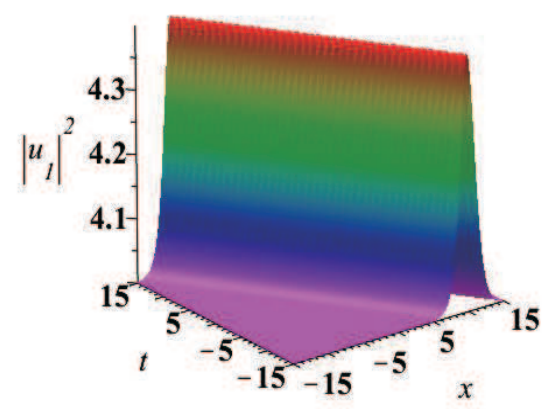

(a) $\left|u_{1}\right|^{2}$

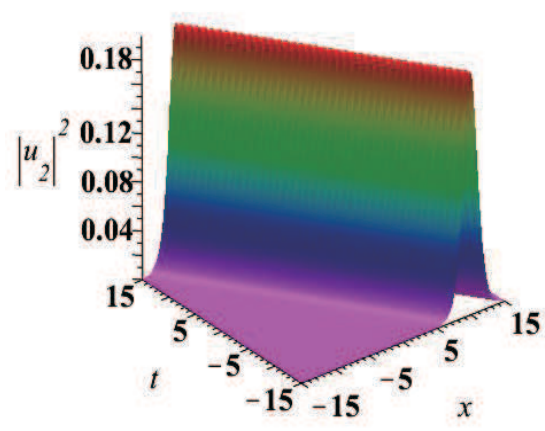

(b) $\left|u_{2}\right|^{2}$

FIG. 4: (color online): Bright-anti-dark soliton solution with parameters: $a_{1}=2, b_{1}=b_{2}=1, z_{1}=\frac{3}{2}-\frac{3}{4} \sqrt{2}$ i, $\kappa_{1}=1-2 \sqrt{2}$, $\kappa_{2}=1+\frac{\sqrt{2}}{2} \mathrm{i}, c_{1}=0, c_{2}=c_{3}=1$. The peaks value for $\left|u_{1}\right|^{2}$ is 4.399 and for $\left|u_{2}\right|^{2}$ is 0.199 .

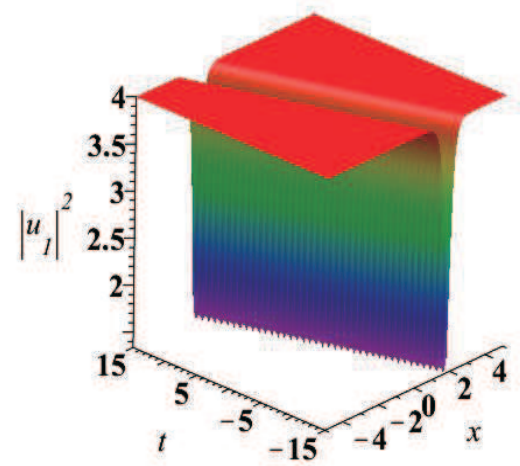

(a) $\left|u_{1}\right|^{2}$

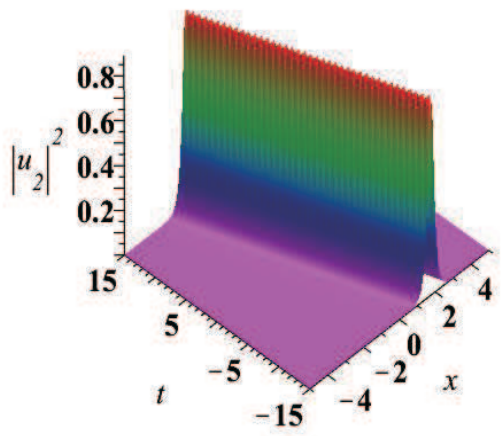

(b) $\left|u_{2}\right|^{2}$

FIG. 5: (color online): Bright-dark soliton solution with parameters: $a_{1}=2, b_{1}=b_{2}=1, z_{1}=\frac{3}{2}-\frac{3}{4} \sqrt{2}$ i, $\kappa_{1}=1-2 \sqrt{2}$, $\kappa_{2}=1+\frac{\sqrt{2}}{2} \mathrm{i}, c_{2}=0, c_{1}=c_{3}=1$. The peaks value for $\left|u_{1}\right|^{2}$ is $\frac{4}{3}$ and for $\left|u_{2}\right|^{2}$ is $\frac{8}{9}$.

Choosing $\lambda_{1} \in \mathbb{R} \cup \mathrm{i}$ such that the characteristic equations (43), (44) possess a pair of conjugate complex roots. Further, by taking a special solution

$$
\left|y_{1}\right\rangle=D_{1}\left[\left(\begin{array}{c}
1 \\
\frac{a_{1} b_{1}}{\lambda_{1}\left(\kappa_{1}+b_{1}\right)} \\
\frac{a_{1} b_{1}}{\lambda_{1}\left(\kappa_{1}+b_{1}\right)}
\end{array}\right) \mathrm{e}^{\vartheta_{1}}+\alpha_{1}\left(\lambda_{1}^{2}-\lambda_{1}^{* 2}\right)\left(\begin{array}{c}
1 \\
\frac{a_{1} b_{1}}{\lambda_{1}\left(\kappa_{2}+b_{1}\right)} \\
\frac{a_{1} b_{1}}{\lambda_{1}\left(\kappa_{2}+b_{1}\right)}
\end{array}\right) \mathrm{e}^{\vartheta_{2}}\right]
$$

and combining with the limit $\lambda_{1} \rightarrow \pm \lambda_{1}^{*}, \kappa_{2} \rightarrow \kappa_{1}^{*}$ for an appropriate $\alpha_{1}$, we obtain

$$
\begin{aligned}
& \beta \rightarrow \frac{2 \lambda_{1}^{-1}}{\kappa_{1}-\kappa_{1}^{*}}\left[\kappa_{1}^{*} \mathrm{e}^{\vartheta_{1}+\vartheta_{1}^{*}} \pm\left|\kappa_{1}\right|\right], \\
& \psi_{1} \varphi_{1}^{*} \rightarrow \frac{a_{1} b_{1} \lambda_{1}^{-1}}{\kappa_{1}+b_{1}} \mathrm{e}^{\vartheta_{1}+\vartheta_{1}^{*}} \\
& \chi_{1} \varphi_{1}^{*} \rightarrow \frac{a_{2} b_{2} \lambda_{1}^{-1}}{\kappa_{1}+b_{1}} \mathrm{e}^{\vartheta_{1}+\vartheta_{1}^{*}} .
\end{aligned}
$$

Finally, we can obtain a soliton solution of either dark or anti-dark type

$$
u_{s}[1]=a_{s}\left[\frac{\kappa_{1} \exp \left(\vartheta_{1}+\vartheta_{1}^{*}+\mathrm{i} \tau_{s}\right)+\varsigma_{1}\left|\kappa_{1}\right|}{\kappa_{1}^{*} \exp \left(\vartheta_{1}+\vartheta_{1}^{*}\right)+\varsigma_{1}\left|\kappa_{1}\right|}\right] \mathrm{e}^{\mathrm{i} \omega_{s}},
$$


where $\exp \left(\mathrm{i} \tau_{s}\right)=\frac{\kappa_{1}^{*}+b_{s}}{\kappa_{1}+b_{s}}$ and $\varsigma_{1}= \pm 1$. The peak values of $\left|u_{s}[1]\right|^{2}$ are

$$
a_{i}^{2}\left[1+\frac{2 b_{i} \kappa_{1 I}^{2}}{\left(\kappa_{1 R}+\varsigma_{1}\left|\kappa_{1}\right|\right)\left|\kappa_{1}+b_{i}\right|^{2}}\right]
$$

. It can be shown by direct calculation that if $\varsigma_{1} b_{i}>0$, the solution (58) is an anti-dark soliton; if $\varsigma_{1} b_{i}>0$, it is a dark soliton. The velocity of the dark/anti-dark soliton is $v=\frac{-1}{b_{1} b_{2} z_{1}}\left(\kappa_{1 R}-z_{1}+\frac{b_{1}+b_{2}}{2}\right)$. To obtain the stationary solution, we must choose the parameters such that $b_{1}+b_{2}=0, \kappa_{1 R}=z_{1}>0, \kappa_{1}=\kappa_{1 R}+\mathrm{i} \kappa_{1 I}$, and

$$
a_{1}= \pm \frac{\sqrt{2}}{2\left|b_{1} \lambda_{1}\right|} \sqrt{\left(b_{1} \lambda_{1}^{2}+1\right)^{2}+\kappa_{1 I}^{2} \lambda_{1}^{4}}, a_{2}= \pm \frac{\sqrt{2}}{2\left|b_{1} \lambda_{1}\right|} \sqrt{\left(b_{1} \lambda_{1}^{2}-1\right)^{2}+\kappa_{1 I}^{2} \lambda_{1}^{4}}
$$

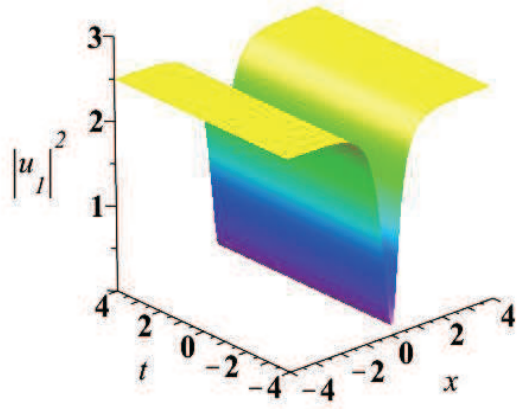

(a) $\left|u_{1}\right|^{2}$

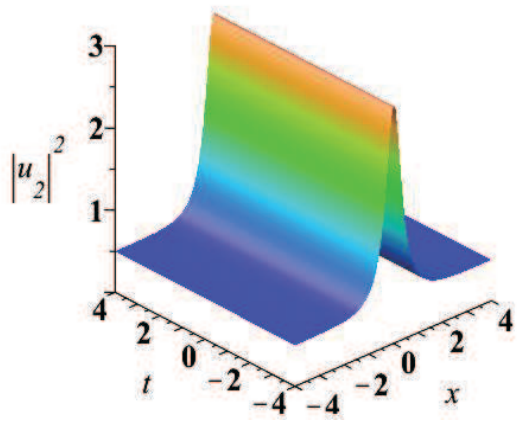

(b) $\left|u_{2}\right|^{2}$

FIG. 6: (color online): Dark-anti-dark soliton solution with parameters: $a_{1}=\frac{\sqrt{2}}{2}, a_{2}=\frac{\sqrt{10}}{2}, b_{1}=-b_{2}=1, \lambda_{1}=1, z_{1}=1$, $\kappa_{1}=1+\mathrm{i}, \kappa_{2}=1-\mathrm{i}$. The peaks value for $\left|u_{1}\right|^{2}$ is 0.085 and for $\left|u_{2}\right|^{2}$ is 2.914 .

If $\kappa_{1}$ is a repeated real root to the characteristic equation (43) or (44), choosing the appropriate parameter $\varsigma_{1}$ in the formula (58), we can obtain the following rational solution through the limit technique

$$
u_{s}[1]=a_{s}\left[\frac{2 \kappa_{1}\left(x+\frac{1}{b_{1} b_{2} z_{1}}\left[\kappa_{1}-z_{1}+\frac{b_{1}+b_{2}}{2}\right] t\right)+\mathrm{i} \frac{\kappa_{1}-b_{s}}{\kappa_{1}+b_{s}}}{2 \kappa_{1}\left(x+\frac{1}{b_{1} b_{2} z_{1}}\left[\kappa_{1}-z_{1}+\frac{b_{1}+b_{2}}{2}\right] t\right)+\mathrm{i}}\right] \mathrm{e}^{\mathrm{i} \omega_{s}} .
$$

It can be easily shown that the peak values for $\left|u_{s}[1]\right|^{2}$ are $a_{s}^{2} \frac{\left(\kappa_{1}-b_{s}\right)^{2}}{\left(\kappa_{1}+b_{s}\right)^{2}}$. What we should point out that the rational solution obtained here is a soliton solution, not a rogue wave solution.

\section{Breather-like solution with nonvanishing boundary condition}

To gain other types of solutions, it is necessary to provide an expression in the numerator of the formula (48). We conclude it by the following proposition.

Proposition 4 If we choose a special solution $\left|y_{1}\right\rangle=\left(\varphi_{1}, \psi_{1}, \chi_{1}\right)^{\mathrm{T}} \equiv \Phi_{2}\left(\lambda_{1}\right)\left(c_{1}, c_{2}, c_{3}\right)^{\mathrm{T}}$, then

$$
\beta+\frac{2 \psi_{1} \varphi_{1}^{*}}{a_{1}} \mathrm{e}^{-\mathrm{i} \omega_{1}}=2 \lambda_{1}^{-1} N_{1}^{[3]}, \beta+\frac{2 \chi_{1} \varphi_{1}^{*}}{a_{2}} \mathrm{e}^{-\mathrm{i} \omega_{2}}=2 \lambda_{1}^{-1} N_{2}^{[3]},
$$

where

$$
N_{s}^{[3]}=K_{l, m}^{[s]}: c_{m} c_{l}^{*} \vartheta^{\vartheta_{m}+\vartheta_{l}^{*}} \equiv \sum_{l, m=1}^{3,3} K_{l, m}^{[s]} c_{m} c_{l}^{*} \mathrm{e}^{\vartheta_{m}+\vartheta_{l}^{*}}, s=1,2
$$

and

$$
\vartheta_{i}=\mathrm{i}\left(\kappa_{i}-z_{1}\right)\left[x+\frac{z_{1}^{*}}{2 b_{1} b_{2}\left|z_{1}\right|^{2}}\left(\kappa_{i}-z_{1}+b_{1}+b_{2}\right) t\right], i=1,2,3 .
$$




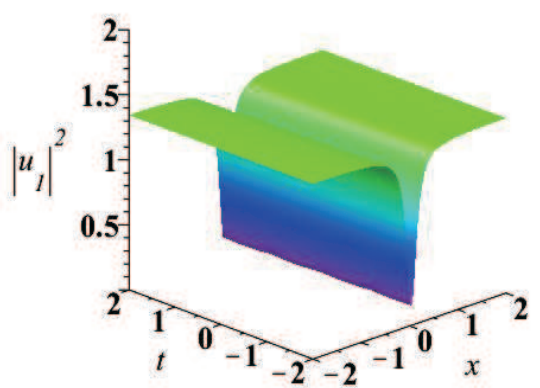

(a) $\left|u_{1}\right|^{2}$

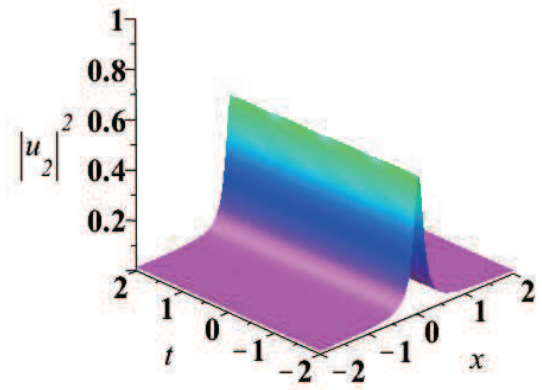

(b) $\left|u_{2}\right|^{2}$

FIG. 7: (color online): Dark-anti-dark soliton solution with parameters: $a_{1}=\frac{3 \sqrt{15}}{10}, a_{2}=\frac{\sqrt{10}}{30}, b_{1}=2, b_{2}=-3, \lambda_{1}=\frac{1}{2}, z_{1}=4$, $\kappa_{1}=4$. The peaks value for $\left|u_{1}\right|^{2}$ is $\frac{3}{20}$ and for $\left|u_{2}\right|^{2}$ is $\frac{49}{90}$.

- If $a_{1}, a_{2}, b_{1}, b_{2} \in \Omega_{1}$ and $a_{2} \neq 0$, then

$$
K_{l, m}^{[i]}= \begin{cases}\frac{\kappa_{l}^{*}+b_{1}}{\kappa_{m}+b_{1}} \frac{\kappa_{m}}{\kappa_{m}-\kappa_{l}^{*}} & \text { if } 1 \leq l \leq 2,1 \leq m \leq 2, l+m<4, \\ \frac{\left|c_{2}\right|^{2} z_{1}^{2}\left(\left(a_{1}^{2}+\sigma a_{2}^{2}\right) z_{1}^{*}-1\right)}{\left|z_{1}\right|^{2}-z_{1}^{2}} & \text { if } l=m=2, \\ \frac{\left(\sigma+\frac{a_{2}^{2}}{a_{1}^{2}}\right)\left|z_{1}\right|}{z_{1}^{*}-z_{1}} & \text { if } l=m=3, \\ -\frac{a_{2}}{a_{1}^{2}} \lambda_{1} & \text { if } l=1,2, m=3, i=1, \\ \frac{\lambda_{1}}{a_{2}} & \text { if } l=1,2, m=3, i=2, \\ 0 & \text { otherwise. }\end{cases}
$$

- If $a_{1}, a_{2}, b_{1}, b_{2} \in \Omega_{2}$, then

$$
K_{l, m}^{[i]}= \begin{cases}\frac{\kappa_{l}^{*}+b_{1}}{\kappa_{m}+b_{1}} \frac{\kappa_{m}}{\kappa_{m}-\kappa_{l}^{*}} & \text { if } 1 \leq l \leq 2,1 \leq m \leq 2, \\ \frac{\left(\sigma+\frac{a_{2}^{2}}{a_{1}^{2}}\right)\left|z_{1}\right|}{z_{1}^{*}-z_{1}} & \text { if } l=m=3, \\ -\frac{a_{2}}{a_{1}^{2}} \lambda_{1} & \text { if } l=1,2, m=3, i=1 \\ \frac{\lambda_{1}}{a_{2}} & \text { if } l=1,2, m=3, i=2 \\ 0 & \text { otherwise. }\end{cases}
$$

- If $a_{1}, a_{2}, b_{1}, b_{2} \in \Omega_{3}$, then

$$
K_{l, m}^{[i]}= \begin{cases}\frac{\left|c_{2}\right|^{2} z_{1}^{2}\left(\left(a_{1}^{2}+\sigma a_{2}^{2}\right) z_{1}^{*}-1\right)}{\left|z_{1}\right|^{2}-z_{1}^{2}}, & \text { if } l=m=3, \\ \frac{\kappa_{l}^{*}+b_{i}}{\kappa_{m}+b_{i}} \frac{\kappa_{m}}{\kappa_{m}-\kappa_{l}^{*}}, & \text { others }\end{cases}
$$

- If $a_{1}, a_{2}, b_{1}, b_{2} \in \Omega_{4}$, then

$$
K_{l, m}^{[i]}=\frac{\kappa_{l}^{*}+b_{i}}{\kappa_{m}+b_{i}} \frac{\kappa_{m}}{\kappa_{m}-\kappa_{l}^{*}} .
$$

Proposition 4 can be proved in the similar way as Proposition 3 thus we omit the proof here. 
Based on Propositions 3 and 4, we can obtain the general solution

$$
u_{s}[1]=a_{s}\left(\frac{N_{s}^{[3]}}{M_{1}}\right) \mathrm{e}^{\mathrm{i} \omega_{s}} .
$$

In what follows, we present the dynamics for the general solution. When $a_{1}, a_{2}, b_{1}, b_{2} \in \Omega_{1} \cup \Omega_{3}$, we have a stationary soliton solution. When $a_{1}, a_{2}, b_{1}, b_{2} \in \Omega_{1}$ and $c_{1} c_{3} \neq 0, c_{2}=0$, then we obtain a very complicated soliton solution, whose dynamics is difficult to be analyzed. The denominator of $\left|u_{s}[1]\right|^{2}$ is

$$
\left|M_{1}\right|^{2}=O\left(1+\frac{\left(z_{1}^{*}-b_{1} / 2\right)\left|c_{1}\right|^{2}}{\left|z_{1}\right|\left(1+\sigma a_{2}^{2} / a_{1}^{2}\right)\left|c_{3}\right|^{2}} \mathrm{e}^{\vartheta_{1}-\vartheta_{3}+\vartheta_{1}^{*}-\vartheta_{3}^{*}}\right),
$$

and the center of this soliton turns out to be

$$
x=\frac{1}{4 \operatorname{Im}\left(z_{1}\right)} \ln \left|\frac{\left(z_{1}^{*}-b_{1} / 2\right)\left|c_{1}\right|^{2}}{\left|z_{1}\right|\left(1+\sigma a_{2}^{2} / a_{1}^{2}\right)\left|c_{3}\right|^{2}}\right| .
$$

When $a_{1}, a_{2}, b_{1}, b_{2} \in \Omega_{3}$ and $c_{1} c_{2} \neq 0, c_{3}=0$, then one has a similar complicated soliton solution, whose center is located at

$$
x=\frac{1}{\delta_{1} \operatorname{Im}\left(\xi_{2} \pm \xi_{2}^{-1}\right)} \ln \left|\frac{\kappa_{1}^{*}\left(\kappa_{2}^{*}-\kappa_{2}\right)\left|c_{1}\right|^{2}}{\kappa_{2}^{*}\left(\kappa_{1}^{*}-\kappa_{1}\right)\left|c_{2}\right|^{2}}\right|,
$$

here $\xi_{2}$ is given in the formula (46).

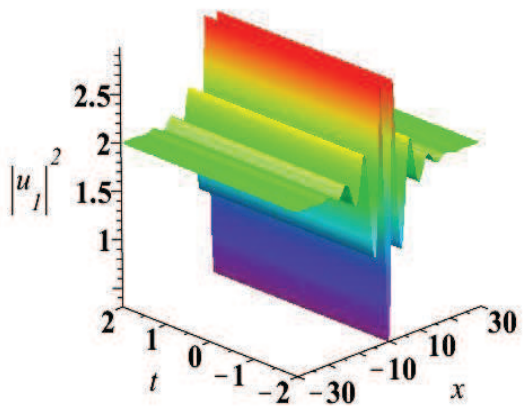

(a) $\left|u_{1}\right|^{2}$

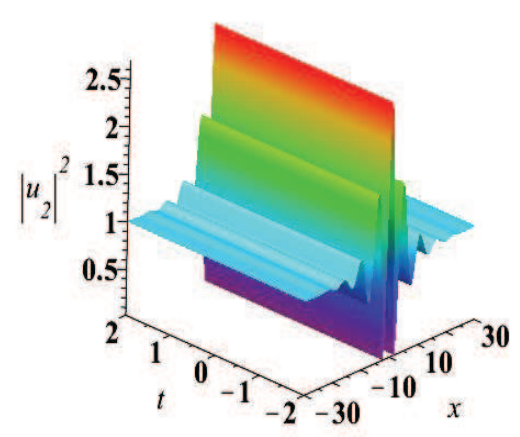

(b) $\left|u_{2}\right|^{2}$

FIG. 8: (color online): A resonant breather solution with parameters: $a_{1}=\sqrt{2}, a_{2}=1, b_{1}=\frac{1}{2}, b_{2}=1, z_{1}=\frac{8}{17}+\frac{9}{68}$ i, $\kappa_{1}=\frac{1}{4}+\frac{1}{4} \mathrm{i}, \kappa_{2}=-\frac{55}{68}+\frac{\mathrm{i}}{68}, \kappa_{3}=0, c_{1}=1, c_{2}=\frac{1}{6}, c_{3}=0$.

Other cases lead to the breather-like solution with nonvanishing boundary condition. Particularly, if $c_{1} c_{2} c_{3} \neq 0$, a resonant breather solution can be obtained (see Fig. 8). Actually, this type of breather solution also exists in the CNLS equation.

\section{MULTI-DARK/ANTI-DARK SOLITON SOLUTIONS}

In this section, we give the muti-dark/anti-dark soliton solution and its asymptotical analysis. To this end, we take a special solution

$$
\begin{aligned}
& \left|y_{i}\right\rangle=D_{1}\left[\left(\begin{array}{c}
1 \\
\frac{a_{1} b_{1}}{\lambda_{i}\left(\kappa_{i, 1}+b_{1}\right)} \\
\frac{a_{1} b_{1}}{\lambda_{i}\left(\kappa_{i, 1}+b_{1}\right)}
\end{array}\right) \mathrm{e}^{\vartheta_{i, 1}}+\alpha_{i}\left(\lambda_{i}^{2}-\lambda_{i}^{* 2}\right)\left(\begin{array}{c}
1 \\
\frac{a_{1} b_{1}}{\lambda_{i}\left(\kappa_{i, 2}+b_{1}\right)} \\
\frac{a_{1} b_{1}}{\lambda_{i}\left(\kappa_{i, 2}+b_{1}\right)}
\end{array}\right) \mathrm{e}^{\vartheta_{i, 2}}\right] \\
& \vartheta_{i, l}=\mathrm{i}\left(\kappa_{i, l}-z_{i}\right)\left(x+\frac{1}{2 b_{1} b_{2} z_{i}}\left(\kappa_{i, l}-z_{i}+b_{1}+b_{2}\right) t\right), l=1,2,
\end{aligned}
$$




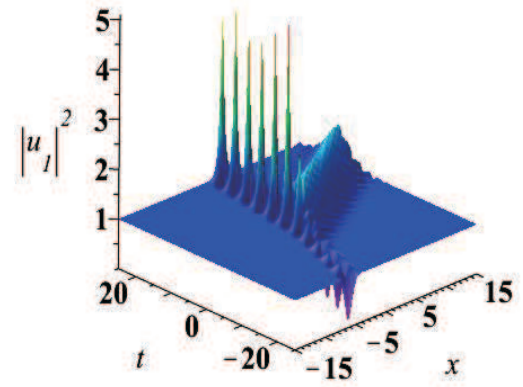

(a) $\left|u_{1}\right|^{2}$

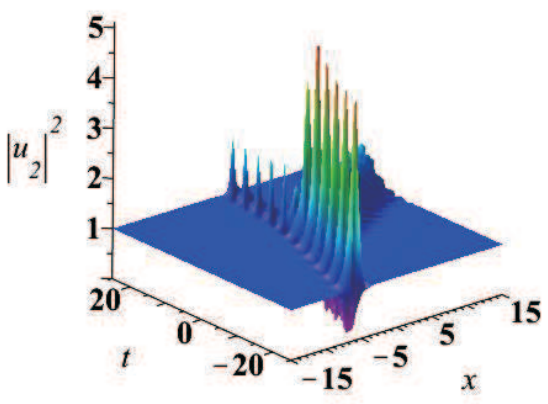

(b) $\left|u_{2}\right|^{2}$

FIG. 9: (color online): Resonant breather solution with parameters: $a_{1}=a_{2}=1, b_{1}=-b_{2}=1, z_{1}=\mathrm{i}, \kappa_{1}=1.359959341-$ $.2573179718 \mathrm{i}, \kappa_{2}=-.7352886592+2.169427339 \mathrm{i}, \kappa_{3}=-.6246706814+0.087890633 \mathrm{i}, c_{1}=1, c_{2}=1, c_{3}=1$.

where $\kappa_{i, l}(l=1,2)$ satisfies the following equation

$$
\left(z_{i}^{-1} \kappa_{i}-2\right)\left(\kappa_{i}+b_{1}\right)\left(\kappa_{i}+b_{2}\right)+a_{1}^{2} b_{1}^{2}\left(\kappa_{i}+b_{2}\right)+\sigma a_{2}^{2} b_{2}^{2}\left(\kappa_{i}+b_{1}\right)=0
$$

It is noted that as $z_{i} \rightarrow z_{i}^{*}, \kappa_{i, 2} \rightarrow \kappa_{i, 1}^{*}$. Based on above equations, one obtains

$$
\begin{aligned}
& M=\left(m_{i j}\right)_{1 \leq i, j \leq N}, \quad m_{i j}=\frac{2 \lambda_{j}^{-1}}{\kappa_{j, 1}-\kappa_{i, 1}^{*}}\left[\kappa_{i, 1}^{*} \mathrm{e}^{\vartheta_{i, 1}+\vartheta_{i, 1}^{*}}+\delta_{i, j} \varsigma_{i}\left|\kappa_{i, 1}\right|\right], \\
& H_{s}=\left(h_{i j}^{[s]}\right)_{1 \leq i, j \leq N}, \quad h_{i j}^{[s]}=\frac{2 \lambda_{j}^{-1}}{\kappa_{j, 1}-\kappa_{i, 1}^{*}}\left[\frac{\kappa_{i, 1}^{*}+b_{s}}{\kappa_{j, 1}+b_{s}} \kappa_{j, 1} \mathrm{e}^{\vartheta_{i, 1}+\vartheta_{i, 1}^{*}}+\delta_{i, j} \varsigma_{i}\left|\kappa_{i, 1}\right|\right],
\end{aligned}
$$

where $\delta_{i, j}$ is the Kronecker's delta, $\varsigma_{i}= \pm 1$. For simplicity, we denote $\kappa_{i, 1}$ as $\kappa_{i}$ and assume $\kappa_{i}=\kappa_{i, R}+\mathrm{i} \kappa_{i, I}$. By tedious calculations, the multi-dark/anti-dark soliton solution can be represented by

$$
u_{s}[N]=a_{s}\left[\frac{\operatorname{det}\left(H_{s}\right)}{\operatorname{det}(M)}\right] \mathrm{e}^{\mathrm{i} \omega_{i}}
$$

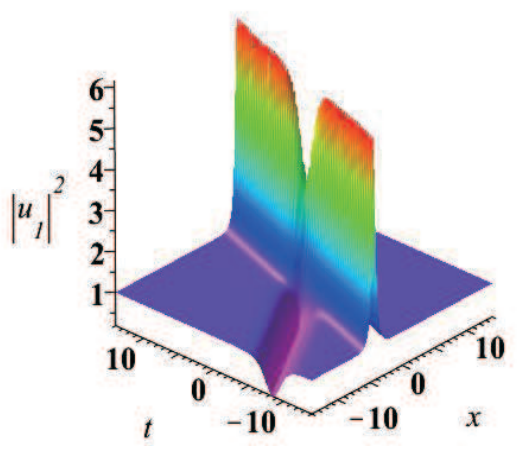

(a) $\left|u_{1}\right|^{2}$

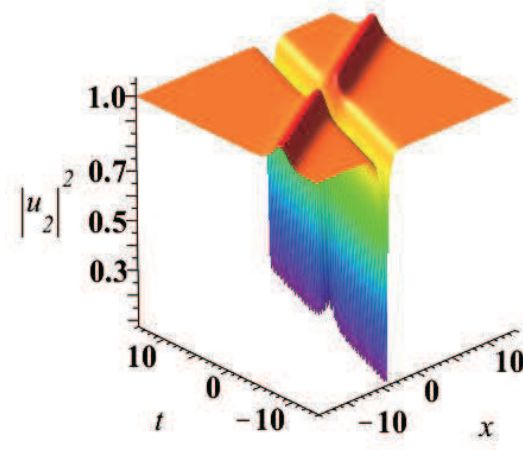

(b) $\left|u_{2}\right|^{2}$

FIG. 10: (color online): Two-dark soliton solution with parameters: $a_{1}=a_{2}=1, b_{1}=-b_{2}=1, \lambda_{1}=1, \lambda_{2}=2, \kappa_{1}=$ $1.347810385-1.028852255 \mathrm{i}, \kappa_{2}=0.6647417703-0.4011272786 \mathrm{i}, \varsigma_{1}=-1, \varsigma_{2}=1$.

In what follows, we perform analysis for the asymptotical behavior of the $N$-dark/anti-dark soliton solution. Introducing the determinant of Cauchy matrix

$$
\Delta_{k} \equiv\left|\frac{1}{\kappa_{j}-\kappa_{i}^{*}}\right|_{1 \leq i, j \leq k},
$$




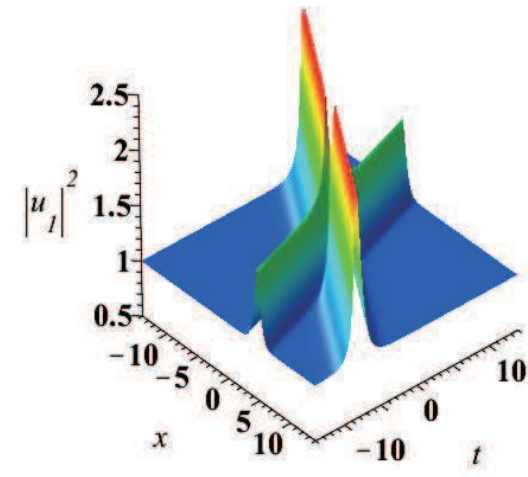

(a) $\left|u_{1}\right|^{2}$

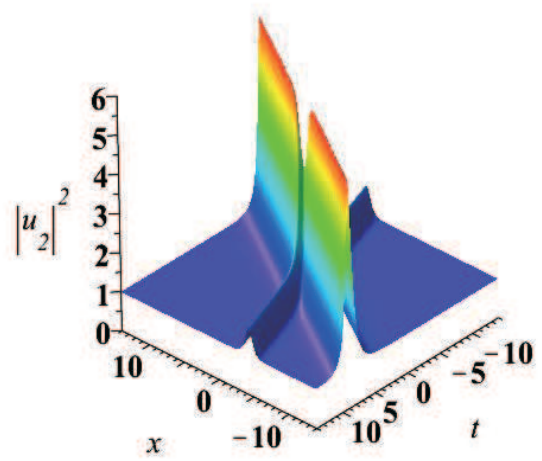

(b) $\left|u_{2}\right|^{2}$

FIG. 11: (color online): Two-dark soliton solution with parameters: $a_{1}=a_{2}=1, b_{1}=2, b_{2}=1, \lambda_{1}=1, \lambda_{2}=2$, $\kappa_{1}=0.1766049822-1.2028208 \mathrm{i}, \kappa_{2}=-0.4486076425-0.3327284758 \mathrm{i}, \varsigma_{1}=1, \varsigma_{2}=1$.

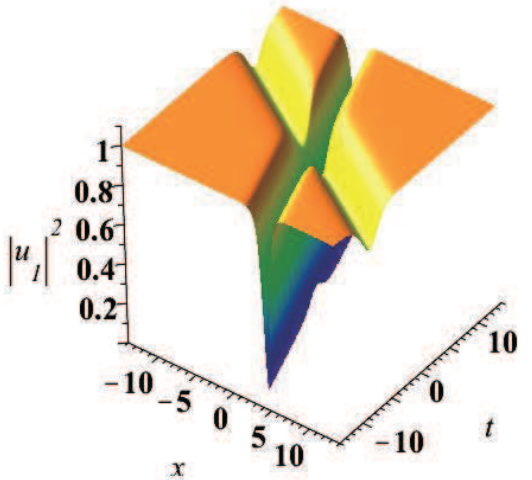

(a) $\left|u_{1}\right|^{2}$

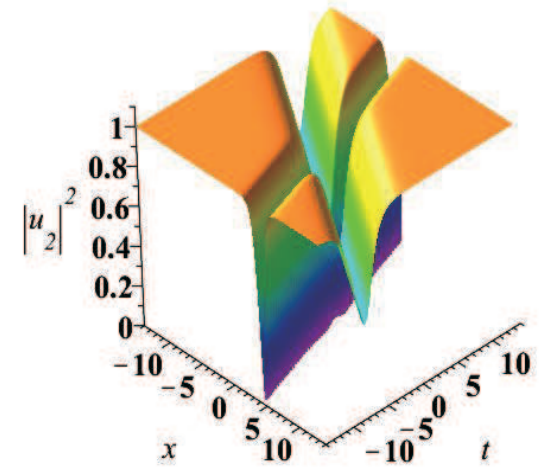

(b) $\left|u_{2}\right|^{2}$

FIG. 12: (color online): Two-dark soliton solution with parameters: $a_{1}=a_{2}=1, b_{1}=2, b_{2}=1, \lambda_{1}=1, \lambda_{2}=2$, $\kappa_{1}=0.1766049822-1.2028208 \mathrm{i}, \kappa_{2}=-0.4486076425-0.3327284758 \mathrm{i}, \varsigma_{1}=-1, \varsigma_{2}=-1$.

the asymptotical behavior of $N$-dark soliton can be concluded by

Proposition 5 As $t \rightarrow \pm \infty, u_{s}[N]$ can be expressed as sum of single dark soliton solutions,

$$
u_{s}[N]=a_{s}\left[S_{1}^{[s] \pm}+\left(S_{2}^{[s] \pm}-A_{1}^{[s] \pm}\right)+\cdots+\left(S_{N}^{[s] \pm}-A_{N-1}^{[s] \pm}\right)\right] \mathrm{e}^{\mathrm{i} \omega_{s}}+O\left(\mathrm{e}^{-c|t|}\right)
$$

where $c=\min _{1,2, \cdots, N}\left(\left|\kappa_{i}\right|\right) \min _{i \neq j}\left(\left|v_{i}-v_{j}\right|\right)$,

$$
S_{k}^{[s] \pm}=P_{k}^{[s] \pm}\left[\frac{\kappa_{k} \exp \left(2 \operatorname{Re}\left(\vartheta_{k}^{ \pm}\right)+\mathrm{i} \tau_{k}^{[s]}\right) \pm\left|\kappa_{k}\right|}{\kappa_{k}^{*} \exp \left(2 \operatorname{Re}\left(\vartheta_{k}^{ \pm}\right)\right) \pm\left|\kappa_{k}\right|}\right], \exp \left(\mathrm{i} \tau_{k}^{[s]}\right)=\frac{\kappa_{k}^{*}+b_{s}}{\kappa_{k}+b_{s}},
$$

and

$$
\begin{aligned}
P_{k}^{[s]-} & =\prod_{i=1}^{k-1}\left(\frac{\left(\kappa_{i}^{*}+b_{s}\right) \kappa_{i}}{\left(\kappa_{i}+b_{s}\right) \kappa_{i}^{*}}\right), P_{k}^{[s]+}=\prod_{i=k+1}^{N}\left(\frac{\left(\kappa_{i}^{*}+b_{s}\right) \kappa_{i}}{\left(\kappa_{i}+b_{s}\right) \kappa_{i}^{*}}\right), \\
\vartheta_{k}^{-} & =\vartheta_{k}+\sum_{i=1}^{k-1} \ln \left|\frac{\kappa_{k}-\kappa_{i}}{\kappa_{k}^{*}-\kappa_{i}}\right|, \vartheta_{k}^{+}=\vartheta_{k}+\sum_{i=k+1}^{N} \ln \left|\frac{\kappa_{k}-\kappa_{i}}{\kappa_{k}^{*}-\kappa_{i}}\right|, \\
A_{i}^{[s]-} & =P_{i}^{[s]-} \mathrm{e}^{\mathrm{i} \tau_{i}} \frac{\kappa_{i}}{\kappa_{i}^{*}}, A_{i}^{[s]+}=P_{i}^{[s]+} .
\end{aligned}
$$


Proof: The determinant $\operatorname{det}\left(H_{s}\right)$ and $\operatorname{det}(M)$ in $N$-dark/anti-dark soliton solution can be represented as

$$
\begin{aligned}
& \operatorname{det}\left(H_{s}\right)=\left|\frac{1}{\kappa_{j}-\kappa_{i}^{*}}\left[\frac{\kappa_{i}^{*}+b_{s}}{\kappa_{j}+b_{s}} \kappa_{j} \mathrm{e}^{\vartheta_{j}+\vartheta_{i}^{*}}+\delta_{i, j} \varsigma_{i}\left|\kappa_{i}\right|\right]\right|_{1 \leq i, j \leq N}, \\
& \operatorname{det}(M)=\left|\frac{1}{\kappa_{j}-\kappa_{i}^{*}}\left[\kappa_{i}^{*} \mathrm{e}^{\vartheta_{j}+\vartheta_{i}^{*}}+\delta_{i, j} \varsigma_{i}\left|\kappa_{i}\right|\right]\right|_{1 \leq i, j \leq N} .
\end{aligned}
$$

As $t \rightarrow-\infty$, we fix the value of $\operatorname{Re}\left(\vartheta_{k}\right)$

$$
\operatorname{Re}\left(\vartheta_{k}\right)=-\kappa_{k, I}\left(x-v_{k} t\right)=\mathrm{const}, v_{k}=-\frac{1}{b_{1} b_{2} z_{k}}\left(\kappa_{k, R}-z_{k}+\frac{b_{1}+b_{2}}{2}\right),
$$

and assume $v_{1}<v_{2}<\cdots<v_{N}$. From $\operatorname{Re}\left(\vartheta_{i}\right)=-\kappa_{i, I}\left(x-v_{k} t+\left(v_{k}-v_{i}\right) t\right)$, it is obvious that $\operatorname{Re}\left(\vartheta_{i}\right) \rightarrow+\infty$ for $1 \leq i \leq k-1$ and $\operatorname{Re}\left(\vartheta_{i}\right) \rightarrow-\infty$ for $k+1 \leq i \leq N$. It follows that

$$
\begin{aligned}
\operatorname{det}(M) & =\mathrm{e}^{2 \operatorname{Re}\left(\vartheta_{1}+\vartheta_{2}+\cdots+\vartheta_{k-1}\right)}\left[\operatorname{det}\left(M_{k}\right)+O\left(\mathrm{e}^{-c|t|}\right)\right], \\
\operatorname{det}\left(H_{s}\right) & =\mathrm{e}^{2 \operatorname{Re}\left(\vartheta_{1}+\vartheta_{2}+\cdots+\vartheta_{k-1}\right)}\left[\operatorname{det}\left(H_{k}^{[s]}\right)+O\left(\mathrm{e}^{-c|t|}\right)\right],
\end{aligned}
$$

where

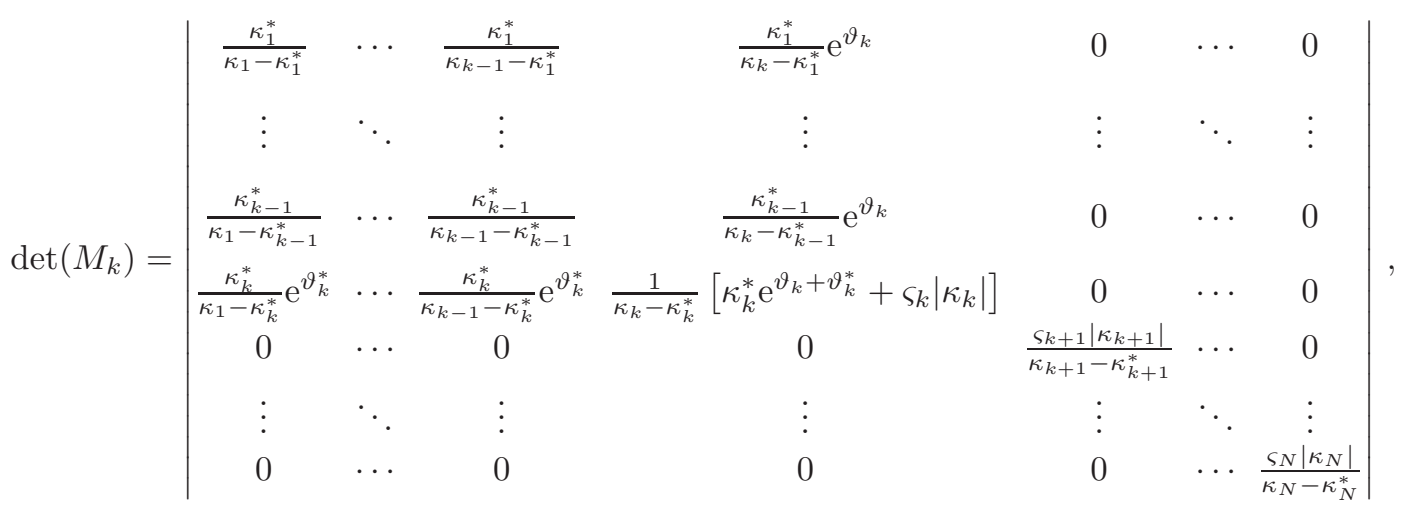

and

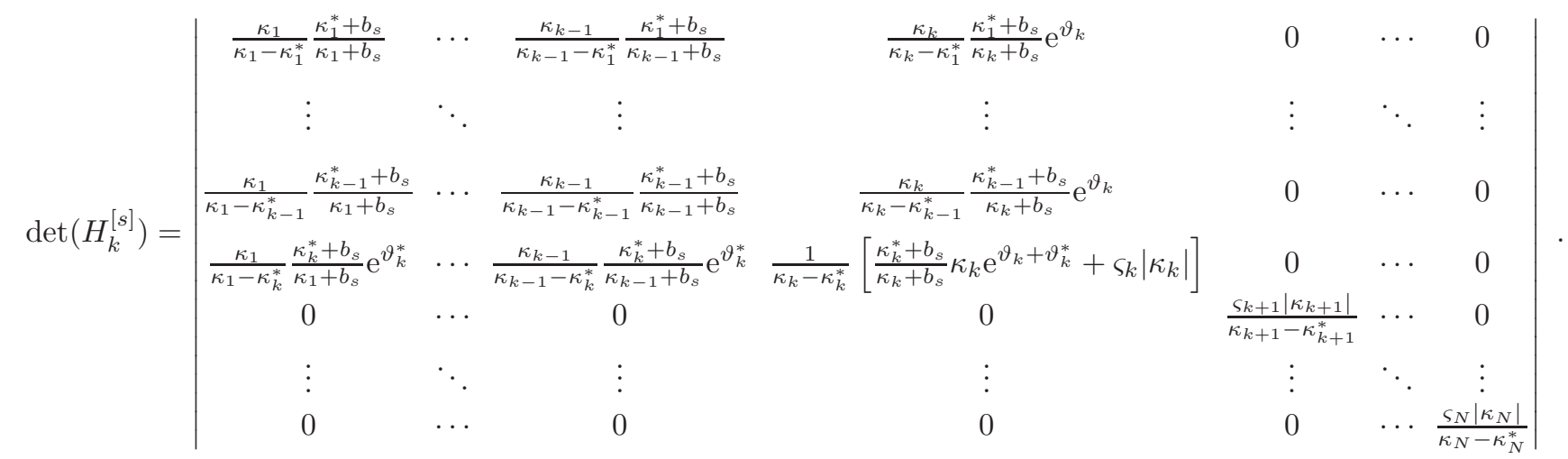

Consequently, as $t \rightarrow-\infty$, we have the asymptotic behavior along $\omega_{l}$ as follows

$$
\begin{aligned}
& u_{s}[N]=a_{s} \prod_{i=1}^{k-1}\left(\frac{\left(\kappa_{i}^{*}+b_{s}\right) \kappa_{i}}{\left(\kappa_{i}+b_{s}\right) \kappa_{i}^{*}}\right)\left[\frac{\frac{\varsigma_{k}\left|\kappa_{k}\right| \Delta_{k-1}}{\kappa_{k}-\kappa_{k}^{*}}+\frac{\kappa_{k}^{*}+b_{s}}{\kappa_{k}+b_{s}} \Delta_{k} \kappa_{k} \mathrm{e}^{\vartheta_{k}^{*}+\vartheta_{k}}}{\frac{\varsigma_{k}\left|\kappa_{k}\right| \Delta_{k-1}}{\kappa_{k}-\kappa_{k}^{*}}+\Delta_{k} \kappa_{k}^{*} \mathrm{e}^{\vartheta_{k}^{*}+\vartheta_{k}}}\right] \mathrm{e}^{\mathrm{i} \omega_{s}}+O\left(\mathrm{e}^{-c|t|}\right) \\
& =a_{s} \prod_{i=1}^{k-1}\left(\frac{\left(\kappa_{i}^{*}+b_{s}\right) \kappa_{i}}{\left(\kappa_{i}+b_{s}\right) \kappa_{i}^{*}}\right)\left[\frac{\varsigma_{k}\left|\kappa_{k}\right|+\prod_{i=1}^{k-1}\left|\frac{\kappa_{k}-\kappa_{i}}{\kappa_{k}^{*}-\kappa_{i}}\right|^{2} \frac{\kappa_{k}^{*}+b_{s}}{\kappa_{k}+b_{s}} \kappa_{k} \mathrm{e}^{\vartheta_{k}^{*}+\vartheta_{k}}}{\varsigma_{k}\left|\kappa_{k}\right|+\prod_{i=1}^{k-1}\left|\frac{\kappa_{k}-\kappa_{i}}{\kappa_{k}^{*}-\kappa_{i}}\right|^{2} \kappa_{k}^{*} \mathrm{e}^{\vartheta_{k}^{*}+\vartheta_{k}}}\right] \mathrm{e}^{\mathrm{i} \omega_{s}}+O\left(\mathrm{e}^{-c|t|}\right) \\
& =a_{s} S_{k}^{-} \mathrm{e}^{\mathrm{i} \omega_{s}}+O\left(\mathrm{e}^{-c|t|}\right),
\end{aligned}
$$


where the relation $\frac{\Delta_{k}}{\Delta_{k-1}}=\frac{1}{\kappa_{k}-\kappa_{k}^{*}} \prod_{i=1}^{k-1}\left|\frac{\kappa_{k}-\kappa_{i}}{\kappa_{k}^{*}-\kappa_{i}}\right|^{2}$ is used.

Similarly, we can prove the asymptotical behavior as $t \rightarrow \infty$, which is omitted here. The proof is complete.

\section{CONCLUSION AND DISCUSSIONS}

In this work, we have constructed the multi-Hamiltonian structure for a multi-component Kaup-Newell hierarchy and the infinite conservation laws for a vector Fokas-Lenells equation. These properties confirm that the vector FL equation is integrable.

Then a generalized Darboux transformation for the coupled FL equation is constructed. By using the DT method, the soliton solutions to the coupled FL equation are thoroughly investigated. Starting from the zero solution, the multi-bright soliton solution is constructed and the analysis of its asymptotic behaviour is performed. On the other hand, starting from a general nonzero seed solution, we have derived a variety of single soliton solutions including the bright-dark soliton, the bright-anti-dark soliton, the dark-dark soliton, the dark-anti-dark soliton and the antidark-anti-dark soliton solutions. Particularly, a breather-like solution with nonvanishing boundary condition is also obtained. In the last, multi-dark solution is deduced by a limit technique developed by one of the authors. The asymptotic behaviour is also analyzed. We should point out that, based on the DT and the plane wave seed solution, one can obtain the higher order rogue wave solutions. Since there are several parameters governing the dynamics of rogue wave, the general rogue wave solution for the coupled FL equation need to analyzed carefully. We would like to report the results in a separate work.

\section{Acknowledgments}

This work is partially supported by National Natural Science Foundation of China (Nos. 11401221, 11428102,11671255) and by the Ministry of Economy and Competitiveness of Spain under contract MTM2012-37070 and MTM2016-80276-P.

[1] A. Shabat, V. Zakharov, Exact theory of two-dimensional self-focusing and one-dimensional self-modulation of waves in nonlinear media, Sov. Phys. JETP 34 (1972) 62-69.

[2] D.J. Kaup, A.C. Newell, An exact solution for a derivative nonlinear Schrödinger equation, J. Math. Phys. 19 (1978) 798-801.

[3] H.H.Chen, Y.C. Lee, C.S. Liu, Integrability of nonlinear Hamiltonian systems by inverse scattering method, Phys. Scr. 20 (1979) 490-492.

[4] V.S. Gerdjikov, M.I. Ivanov, The quadratic bundle of general form and the nonlinear evolution equations, Bulg. J. Phys. 10 (1983) 130-145.

[5] J. Lenells, A.S. Fokas, On a novel integrable generalization of the nonlinear Schrödinger equation, Nonlinearity 22 (2008) 11-27.

[6] J. Lenells, Exactly solvable model for nonlinear pulse propagation in optical fibers, Stud. Appl. Math. 123 (2009) $215-232$.

[7] P. Zhao, E. Fan, Y. Hou, Algebro-geometric solutions and their reductions for the Fokas-Lenells hierarchy, J. Nonl. Math. Phys. 20 (2013) 355-393.

[8] S. Chen, L.Y. Song, Peregrine solitons and algebraic soliton pairs in Kerr media considering space-time correction Phys. Lett. A, 378 (2014) 1228-1232.

[9] S. Xu, J. He, Y. Cheng, K. Porseizan, The n-th order rogue waves of Fokas-Lenells equation, Math. Methods Appl. Sci. 38 (2015) 1106-1126.

[10] Y. Matsuno, A direct method of solution for the Fokas-Lenells derivative nonlinear Schrödinger equation: I. Bright soliton solutions, J. Phys. A: Math. Theor. 45 (2012) 235202.

[11] Y. Matsuno, A direct method of solution for the Fokas-Lenells derivative nonlinear Schrödinger equation: II. Dark soliton solutions J. Phys. A: Math. Theor.45 (2012) 475202.

[12] V.E. Vekslerchik, Lattice representation and dark solitons of the Fokas-Lenells equation, Nonlinearity 24 (2011) 1165-1175.

[13] Y. Zhang, J. W. Yang, K. W. Chow, C. F. Wu, Solitons, breathers and rogue waves for the coupled FokasCLenells system via Darboux transformation, Nonlinear Anal-Real. 332017 237-252.

[14] X.J. Chen, W.K. Lam, Inverse scattering transform for the derivative nonlinear Schrödinger equation with nonvanishing boundary conditions Phys. Rev. E 69 (2004) 066604.

[15] X. J. Chen, J. Yang, Direct perturbation theory for solitons of the derivative nonlinear Schrödinger equation and the modified nonlinear Schrödinger equation Phys. Rev. E 65 (2002) 066608. 
[16] J. Lenells, The solution of the global relation for the derivative nonlinear Schrödinger equation on the half-line Physica D 240 (2011) 512-525.

[17] W.X. Ma, Q. Ding, W.G. Zhang, B.Q. Lu, Binary non-linearization of Lax pairs of Kaup-Newell soliton hierarchy, Nuov. Cim. B 111 (1996)1135-1149.

[18] H. Steudel, The hierarchy of multi-soliton solutions of the derivative nonlinear Schrödinger equation, J. Phys. A 36 (2003 1931-1946.

[19] Y. Xiao, Note on the Darboux transformation for the derivative nonlinear Schrödinger equation, J. Phys. A 24 (1991) 363-366.

[20] V. E. Vekslerchik, Bäcklund transformations between the AKNS and DNLS hierarchies, J. Phys. A: Math. Theor. 44 (2011) 465207.

[21] B. Guo, L. Ling, Q. P. Liu, High-Order Solutions and Generalized Darboux Transformations of Derivative Nonlinear Schrödinger Equations, Stud. in Appl. Math. 130 (2013) 317-344.

[22] X. Y. Wen, Y. Yang, Z. Yan, Generalized perturbation (n, M)-fold Darboux transformations and multi-rogue-wave structures for the modified self-steepening nonlinear Schrödinger equation, Phys. Rev. E 92 (2015) 012917.

[23] X. Lü, M. Peng, Systematic construction of infinitely many conservation laws for certain nonlinear evolution equations in mathematical physics, Commun. Nonlinear Sci. Numer. Simul. 18 (2013) 2304-2312.

[24] X. Lü, Madeling fluid description on a generalized mixed nonlinear Schrödinger equation Nonl. Dyn. 81 (2015) 239-247.

[25] T. Tsuchida, New reductions of integrable matrix partial differential equations: Sp (m)-invariant systems J. Math. Phys. 51 (2010) 053511.

[26] T. Tsuchida, M. Wadati, New integrable systems of derivative nonlinear Schrödinger equations with multiple components, Phys. Lett. A 257 (1999) 53-64.

[27] A. P. Fordy, Derivative nonlinear Schrödinger equations and Hermitian symmetric spaces, J. Phys. A 17 (1984) $1235-1246$.

[28] B. Guo, L. Ling, Riemann-Hilbert approach and N-soliton formula for coupled derivative Schrödinger equation, J. Math. Phys. 53 (2012) 073506.

[29] H.C. Morris, R.K. Dodd R K, The two component derivative nonlinear Schrödinger equation, Phys. Scr. 20 (1979) 505-508.

[30] L. Ling, Q. P. Liu, Darboux transformation for a two-component derivative nonlinear Schrödinger equation, J. Phys. A: Math. Theor. 43 (2010) 434023.

[31] S. V. Manakov, On the theory of two-dimensional stationary self-focusing of electromagnetic waves, Sov. Phys. JETP $38(1974) 248-253$.

[32] M.X. Zhang, A vector Fokas-Lenells system from the coupled Nonlinear Schrödinger Equations, J. Nonlinear Math. Phys. $22(2015) 144 \mathrm{C} 154$.

[33] V. B. Matveev, M. A. Salle, Darboux transformations and solitons, Springer, Berlin, 1991.

[34] C.H. Gu, H.S. Hu, Z.X. Zhou, Darboux transformations in integrable systems: theory and their applications to geometry. Springer 2006.

[35] C.L.Terng, K.Uhlenbeck, Bäcklund transformations and loop group actions, Commun. Pure Appl. Math. 53 (2000) 1-75.

[36] L. Ling, L.C. Zhao, B. Guo, Darboux transformation and classification of solution for mixed coupled nonlinear Schrödinger equations, Commun. Nonlinear Sci. Numer. Simul. 32 (2016) 285-304.

[37] L. Ling, L.C. Zhao, B. Guo, Darboux transformation and multi-dark soliton for N-component nonlinear Schrödinger equations, Nonlinearity 28 (2015) 3243.

[38] D. Bian, B. Guo, L. Ling, High-Order Soliton Solution of Landau-Lifshitz Equation, Stud. in Appl. Math. 134 (2015) 181-214.

[39] L. Ling, B.-F. Feng, Z. Zhu, Multi-soliton, multi-breather and higher-order rogue wave solutions to the complex short pulse equation, Physica D 327 (2016) 13-29.

[40] B.-F. Feng, L. Ling, Z. Zhu, A defocusing complex short pulse equation and its multi-dark soliton solution by Darboux transformation, Phys. Rev. E 93 (2016) 052227.

[41] G. Tu, The trace identity, a powerful tool for constructing the Hamiltonian structure of integrable systems, J. Math. Phys. 30 (1989) 330-338.

[42] T. Xia, E. Fan, The multicomponent generalized Kaup-Newell hierarchy and its multicomponent integrable couplings system with two arbitrary functions, J. Math. Phys. 46 (2005) 46043510.

[43] E. Fan, Integrable evolution systems based on Gerdjikov-Ivanov equations, bi-Hamiltonian structure, finite-dimensional integrable systems and N-fold Darboux transformation, J. Math. Phys. 41 (2000) 7769-7782. 